\title{
Flux- and volume-limited groups/clusters for the SDSS galaxies: catalogues and mass estimation ${ }^{\star}$
} \author{
M. Einasto ${ }^{1}$, and E. Saar ${ }^{1,5}$ \\ 1 Tartu Observatory, Observatooriumi 1, 61602 Tõravere, Estonia \\ e-mail: elmo.tempel@to.ee \\ 2 National Institute of Chemical Physics and Biophysics, Rävala pst 10, 10143 Tallinn, Estonia \\ 3 Leibniz-Institut für Astrophysik Potsdam, an der Sternwarte 16, 14482 Potsdam, Germany \\ ${ }^{4}$ Institute of Physics, University of Tartu, 51010 Tartu, Estonia \\ 5 Estonian Academy of Sciences, Kohtu 6, 10130 Tallinn, Estonia
}

E. Tempel ${ }^{1,2}$, A. Tamm ${ }^{1}$, M. Gramann ${ }^{1}$, T. Tuvikene ${ }^{1,3}$, L. J. Liivamägi ${ }^{1,4}$, I. Suhhonenko ${ }^{1}$, R. Kipper ${ }^{1,4}$

Received 6 February 2014 / Accepted 21 April 2014

ABSTRACT

\begin{abstract}
We provide flux- and volume-limited galaxy group and cluster catalogues based on the spectroscopic sample of the galaxies of SDSS data release 10. We used a modified friends-of-friends method with a variable linking length in the transverse and radial directions to identify as many realistic groups as possible. The flux-limited catalogue incorporates galaxies down to $m_{r}=17.77$ mag. It includes 588193 galaxies and 82458 groups. The volume-limited catalogues are complete for absolute magnitudes down to $M_{r, \text { lim }}=-18.0$, $-18.5,-19.0,-19.5,-20.0,-20.5$, and -21.0 ; the completeness is achieved within different spatial volumes. Our analysis shows that flux- and volume-limited group samples are well compatible, especially for the larger groups/clusters. Dynamical mass estimates based on radial velocity dispersions and group extent in the sky were added to the extracted groups.
\end{abstract}

Key words. galaxies: clusters: general - galaxies: groups: general - galaxies: statistics - large-scale structure of Universe cosmology: observations - catalogs

\section{Introduction}

Galaxies, like stars or people, tend to gather in pairs, groups of several members, or even larger conglomerates. It is only natural that an individual surrounded by companions follows a somewhat different evolutionary path and develops a different appearance and inner qualities than a loner; the dependence of galaxy properties on the environment was shown already decades ago (Einasto et al. 1974; Oemler 1974; Davis \& Geller 1976; Dressler 1980; Postman \& Geller 1984). Therefore, catalogues of galaxy groups and clusters provide an unlimited data source for a wide range of astrophysical and cosmological applications, as illustrated below with just a random pick of the latest studies.

Among the most popular applications, galaxy group and cluster catalogues are used for quantifying the neighbourhood of galaxies in studies of the environmental dependencies of galaxy properties. The published catalogues have enabled a multitude of studies about the dependencies of the morphology, structure, gas content, star formation rate, merger rate, etc. of galaxies on their local environment (some most recent examples include Tempel et al. 2009; Carollo et al. 2013; Catinella et al. 2013; Hess \& Wilcots 2013; Kaviraj 2014; Lackner \& Gunn 2013; Peng \& Maiolino 2014).

In addition, group and cluster catalogues can be used to distinguish central galaxies from their satellites (Lacerna et al. 2014; Wetzel et al. 2013; Yang et al. 2013; Li et al. 2014).

* The group catalogues are available at http://cosmodb. to.ee and at the CDS via anonymous ftp to cdsarc.u-strasbg. fr $(130.79 .128 .5)$ or via

http://cdsarc.u-strasbg.fr/viz-bin/qcat?J/A+A/566/A1
They also simplify the selection and studying of the properties of specific galaxy systems, be it galaxy pairs, compact groups, loose groups, etc. Assuming that galaxy groups and clusters inhabit common dark matter haloes, catalogues can be used to probe dark matter haloes of different mass and to seek correlations between the mass and the galaxy content of the haloes (Carollo et al. 2013; Hou et al. 2013; Huertas-Company et al. 2013; McGee 2013; Wetzel et al. 2013; Yang et al. 2013), or to study the general group properties as a function of group mass (Hearin et al. 2013; Budzynski et al. 2014) or as a function of the large-scale environment (Luparello et al. 2013; Einasto et al. 2014). By comparing the properties of observed groups and clusters with those produced in simulations, our understanding of structure formation can be validated (Nurmi et al. 2013).

Yet another broad field of applications for group and cluster catalogues is their usage for mapping the underlying cosmic web, for example by extracting large-scale filaments (Zhang et al. 2013; Alpaslan et al. 2014), or the largest known density enhancements, galaxy superclusters (Zucca et al. 1993; Einasto et al. 1994, 2001), from the spatial distribution of groups and clusters. Furthermore, the properties of groups in superclusters, and galaxies in them, can be studied (Einasto et al. 2003, 2008, 2011, 2012; Lietzen et al. 2012; Krause et al. 2013).

From the examples above, it is evident that catalogues of galaxy groups and clusters are in demand. But how to define a galaxy group and how to delineate it from galaxy redshift survey data? No straightforward recipe exists, different approaches would be valid for different science goals. In simulations we can see that gravitationally bound galaxy systems are linked by an underlying dark matter halo, thus a good approach for defining a galaxy group or cluster would be through the existence of a 
common dark matter halo. However, for observational datasets, this method is of very little practical value. Instead, the friendsof-friends (FoF) algorithm has remained the most frequently applied means of identifying groups and clusters in galaxy redshift data ever since its introduction (Turner \& Gott 1976; Press \& Davis 1982). Today, many extensive FoF group and cluster catalogues are available, varying in the sample data and details of the group-finder algorithm (e.g. Tucker et al. 2000; Eke et al. 2004; Tago et al. 2006, 2008).

The FoF method uses galaxy distances as the main basis of grouping, and is thus relatively simple and straightforward, while the membership of the produced groups is rather uncertain. Thus several other techniques for group and cluster extraction have been developed, well reviewed by Gal (2006) and recently applied by Yang et al. (2005), Koester et al. (2007), Yang et al. (2007), Hao et al. (2010), Makarov \& Karachentsev (2011), Wen et al. (2012), and Muñoz-Cuartas \& Müller (2012). However, whether any method is more reliable than the others is still largely a matter of taste and debate (Old et al. 2014).

In Tempel et al. (2012), we constructed a flux-limited FoF group and cluster catalogue for galaxies in the Sloan Digital Sky Survey (SDSS, York et al. 2000) data release 8 (DR8; Aihara et al. 2011). Here we present an update of this catalogue, based on SDSS DR10 (Ahn et al. 2014). In addition to a flux-limited catalogue, we present volume-limited catalogues, valid within different galactic absolute luminosity bins (and thus being complete within different spatial volumes). We provide dynamical mass estimates for the detected galaxy systems, using the measured radial velocities and group extent in the sky. The catalogues also contain a rough estimate of the expected total luminosity of each group, assuming that some of the group members are not included in the sample because of observational limitations.

Throughout this paper we assume the Wilkinson Microwave Anisotropy Probe (WMAP) cosmology: the Hubble constant $H_{0}=100 \mathrm{hm} \mathrm{s}^{-1} \mathrm{Mpc}^{-1}$, the matter density $\Omega_{\mathrm{m}}=0.27$ and the dark energy density $\Omega_{\Lambda}=0.73$ (Komatsu et al. 2011).

\section{Data}

\subsection{SDSS main sample}

The present work is based on the SDSS DR10 (York et al. 2000; Ahn et al. 2014). We have used only the main contiguous area of the survey (the Legacy Survey). Since the survey edges in the sky are noisy in some regions, we applied the sample mask as defined by Martínez et al. (2009). Figure 1 shows the SDSS main footprint in the sky, covering 7221 square degrees $(17.5 \%$ from the full sky).

Our previous galaxy group catalogue (Tempel et al. 2012) was based on the SDSS DR8 (Aihara et al. 2011). While the sky coverage of the SDSS main area has remained unchanged already since DR7 (Abazajian et al. 2009), the data quality has improved. The SDSS webpage ${ }^{1}$ lists several small caveats that have been corrected in DR9 (Ahn et al. 2012) and DR10. Most notably, the astrometry of the objects has been corrected in DR9 and the imaging and spectroscopic pipeline have been updated/improved and applied to all images and spectra.

The original data were downloaded from the Catalog Archive Server $\left(\mathrm{CAS}^{2}\right)$ of the SDSS. For the primary selection,

\footnotetext{
1 http://www.sdss3.org/dr10/imaging/caveats.php http://www.sdss3.org/dr10/spectro/caveats.php

2 http://skyserver.sdss3.org/casjobs/
}

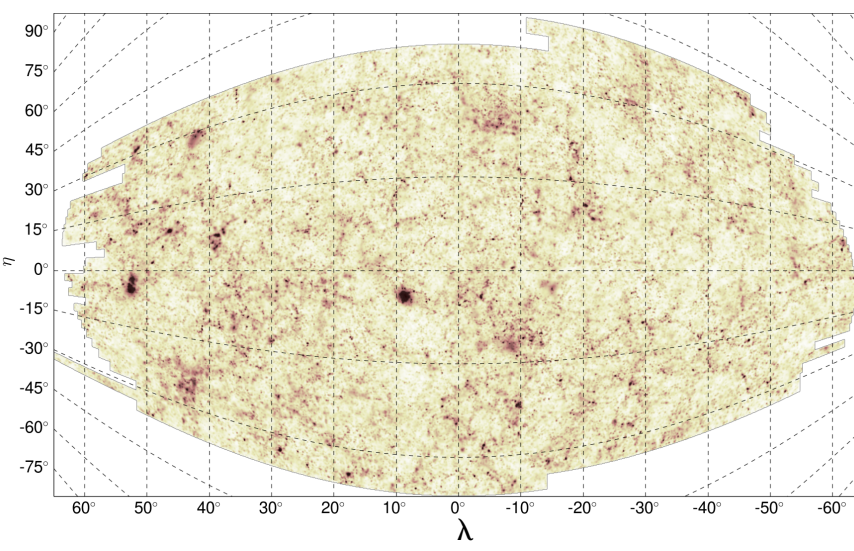

Fig. 1. SDSS main footprint in survey coordinates. Galaxy luminosity density field (integrated over the line of sight) is shown inside a sample mask as defined in Martínez et al. (2009), yielding a sky coverage of 7221 square degrees.

we used the SpecObj table as suggested by the SDSS team for spectroscopic objects. Our first selection included all objects with the spectroscopic class GALAXY or QSO; the final selection of the QSO objects was carried out manually later (see below). The corresponding photometric match was based on bestobjid (position-based match): for objects where it was undefined or the photometric class was not a GALAXY, it was based on fluxobjid (flux-based match). We only used spectroscopic objects for which the final matched photometric object class is GALAXY.

The selected galaxy sample is cleaned and filtered by the SDSS team, but because of the purely automatic filtering, some spurious entries still contaminate the sample. Most notable entries are bright over-saturated stars that are classified as galaxies. Since the luminosity of these objects is high, they would affect the bright-end of the galaxy luminosity function and the luminosity density field. Other spurious entries are large galaxies that sometimes have double/multiple entries in the sample. Moreover, for some of the galaxies, the given luminosity is incorrect because of the proximity of bright stars and/or an incorrect sky estimation. To remove the spurious entires and to flag the galaxies where the luminosity is untrustworthy, we visually checked approximately 30000 galaxies using the SDSS Image List Tool ${ }^{3}$. The steps that were taken to visually clean the sample are described below. In each step, we removed spurious entries and flagged galaxies where the luminosity was obviously incorrect. We checked

- 10000 apparently brightest galaxies (in $r$-band). For galaxies brighter than $m_{r}<13.5$ about $10 \%$ of the objects were spurious. For galaxies $13.5<M_{r}<14.5$, about $1 \%$ were spurious entries; this fraction decreases with luminosity;

- 5000 intrinsically brightest galaxies in the sample $(<1 \%$ were spurious);

- 3000 intrinsically faintest galaxies in the sample (to ensure the correctness of the faint end of the luminosity function);

- all the sources with the spectroscopic class QSO;

- all the objects with bestobjid missing or not GALAXY; for these objects, we used fluxobjid if the matched photometric object was classified as a galaxy;

- all the objects for which the difference between $r$-band point spread function (PSF) magnitude and model magnitude was

3 http://skyserver.sdss3.org/dr10/en/tools/chart/ listinfo.aspx 
smaller than 0.25 (thus further excluding some of the stellar sources in the catalogue);

- all the galaxies with the difference between $r$-band Petrosian and model magnitudes greater than 0.4 ;

- the entries where the colour indices $g-r, r-i$, and $g-i$ had extreme values;

- all the galaxy pairs that were closer than $5^{\prime}$ and had roughly equal redshift (to remove double/multiple entries). This concerns mostly large nearby galaxies.

Altogether, about 600 entries were removed from the initial sample. In the final sample, the number of galaxies with incorrect luminosity (flagged entries in the final catalogue) is 1352 .

After the visual cleaning, we filtered the sample to contain only galaxies with the Galactic extinction corrected (based on Schlegel et al. 1998) Petrosian $r$-band magnitude $m_{r} \leq 17.77$. For fainter objects, the SDSS is incomplete (Strauss et al. 2002). After correcting the redshift relative to the motion with respect to the cosmic microwave background $(\mathrm{CMB})$, we set the upper distance limit at $z=0.2$.

Our galaxy catalogue (see Appendix A) includes all the relevant parameters from the SDSS CAS (apparent magnitudes, coordinates, observed redshift, etc.). To facilitate the use of other SDSS parameters available in CAS, we include the objid and specobjid parameters in our catalogue. Appendix A gives an example of how to query additional parameters from CAS.

In addition to the parameters provided by the SDSS team, we have added several parameters calculated/derived in this paper and explained in Appendix A.

\subsection{Spectroscopically complemented galaxy sample}

The SDSS galaxy sample is not complete, mainly because of the fibre collision - the minimum separation between spectroscopic fibres is $55^{\prime \prime}$. For this reason, about $6 \%$ of galaxies in the SDSS are without observed spectra. In addition, the redshift catalogue is incomplete for bright nearby objects because of the saturation limit of the SDSS detectors. Tempel et al. (2012) studied the effect of missing galaxies on a group catalogue and concluded that the strongest impact is on galaxy pairs. In the SDSS sample, the absent galaxies are more likely to reside in groups and only $4 \%$ of single galaxies lack a companion. The estimated amount of missing pairs in the present group catalogue is about $8 \%$.

To complement the SDSS spectroscopic sample, other redshift surveys covering the same sky area can be used. Following Choi et al. (2010), we used the Two-degree Field Galaxy Redshift Survey (2dFGRS, Colless et al. 2001, 2003), the Two Micron All Sky Survey (2MASS, Jarrett et al. 2003; Skrutskie et al. 2006) Redshift Survey (2MRS, Huchra et al. 2012), and the Third Reference Catalogue of Bright Galaxies (RC3, de Vaucouleurs et al. 1991; Corwin et al. 1994) to complement the SDSS photometric objects with redshifts measured by one of the previously mentioned surveys. The addition of these objects improves the completeness for bright nearby objects and for objects in relatively nearby high-density regions (e.g. the Coma cluster); see Choi et al. (2010) for more details. All these three tables are listed in the SDSS CAS, and we downloaded the data from there.

We matched the SDSS photometric objects with these redshift catalogues using the sky coordinates. Only galaxies brighter than $m_{r}=17.77 \mathrm{mag}$ were considered. As before, we searched for duplicate entries. In addition, every matched galaxy was checked visually to avoid spurious entries.

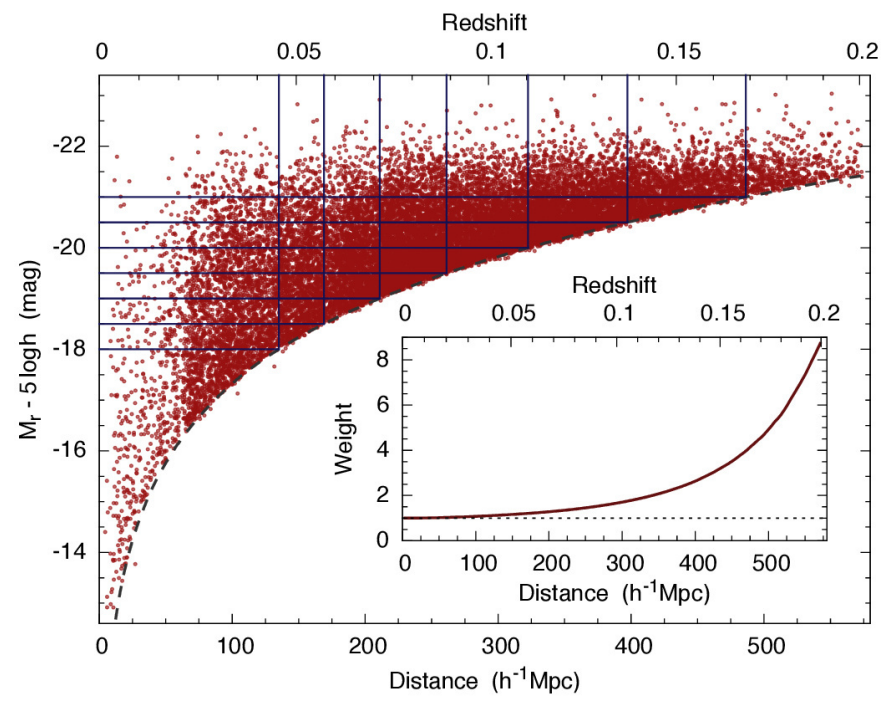

Fig. 2. Galaxy absolute magnitude in the $r$-filter as a function of comoving distance (lower axis) and redshift (upper axis). For visual clarity, only a small fraction of all galaxies is plotted: galaxies are randomly chosen to have approximately uniform number density with distance. The dashed black line shows the upper distance limit for a given absolute magnitude using the average $k$-correction. The solid blue lines show the subsamples for various volume-limited samples. The inset panel shows the luminosity weight factor as a function of distance. This weight takes into account the luminosity that is missed because of the flux-limited survey.

In total, we added 3484 redshifts from the 2dFGRS dataset, 1119 redshifts from the 2 MRS dataset, and 280 redshifts from the RC3. Our final galaxy sample includes 588193 galaxies.

\subsection{Volume-limited galaxy samples}

Intrinsically, the SDSS sample is flux-limited, meaning that the number density of galaxies decreases with distance: only the brightest galaxies are observed farther away (see Fig. 2). However, for many applications (e.g. comparison with simulations, Nurmi et al. 2013), volume-limited samples are desired. In addition to the flux-limited galaxy and group catalogue, we therefore also construct volume-limited galaxy and group catalogues in this paper.

To prepare the volume-limited galaxy sample, we started from the flux-limited galaxy and group samples (Sect. 3.1 gives details for our group-finding method). Due to the peculiar velocities of galaxies in groups, the measured redshift (recession velocity) does not give an accurate distance to a galaxy located in a group/cluster (Jackson 1972). Therefore, the apparent magnitude $m$ was transformed into the absolute magnitude $M$ (corresponding to redshift $z=0$ ) using the group/cluster mean redshift $z_{\mathrm{cl}}$ and mean comoving distance $d_{\text {com }}$ (for isolated galaxies, we used the galaxy redshift and distance),

$M_{\lambda}=m_{\lambda}-25-5 \log _{10}\left(d_{\mathrm{L}}\right)-K_{\lambda}$,

where $d_{\mathrm{L}}=d_{\mathrm{com}}\left(1+z_{\mathrm{cl}}\right)$ is the luminosity distance in units $h^{-1} \mathrm{Mpc}, K_{\lambda}$ is the $k+e$-correction, and the index $\lambda$ refers to each of the ugriz filters. The $k$-corrections were calculated with the KCORRECT (v4_2) algorithm (Blanton \& Roweis 2007). Evolution correction was estimated similarly by Blanton et al. (2003) assuming a distance-independent luminosity function. The estimation of the luminosity evolution is described in Appendix B. 
Table 1. SDSS main sample used for the flux- and volume-limited samples.

\begin{tabular}{|c|c|c|c|c|c|c|c|c|c|c|c|}
\hline Sample & $\begin{array}{c}M_{\mathrm{r}, \mathrm{lim}} \\
\mathrm{mag} \\
1\end{array}$ & $z_{\lim }$ & $\begin{array}{c}d_{\lim } \\
h^{-1} \mathrm{Mpc} \\
3\end{array}$ & $\begin{array}{c}N_{\text {gal }} \\
4\end{array}$ & $N_{\text {groups }}$ & $\begin{array}{c}d_{\text {mean }} \\
h^{-1} \mathrm{Mpc} \\
6\end{array}$ & $\begin{array}{c}L L \\
h^{-1} \mathrm{Mpc} \\
7\end{array}$ & $\begin{array}{c}b_{\text {gal }} \\
8\end{array}$ & $\begin{array}{c}\text { in groups } \\
\% \\
9\end{array}$ & $\begin{array}{c}\text { \# den } \\
h^{3} \mathrm{Mpc}^{-3} \\
10\end{array}$ & Weight \\
\hline Flux-limited & $17.77^{a}$ & 0.200 & 574.2 & 588193 & 82458 & - & $0.23-0.5$ & - & 47.9 & - & - \\
\hline Vol-lim-18.0 & -18.0 & 0.045 & 135.0 & 49860 & 7328 & 3.294 & 0.380 & 0.115 & 59.8 & $4.11 \times 10^{-3}$ & 1.135 \\
\hline Vol-lim-18.5 & -18.5 & 0.057 & 168.9 & 73006 & 10929 & 3.638 & 0.411 & 0.113 & 58.3 & $3.11 \times 10^{-3}$ & 1.204 \\
\hline Vol-lim-19.0 & -19.0 & 0.071 & 211.0 & 105041 & 15715 & 4.027 & 0.445 & 0.110 & 55.7 & $2.29 \times 10^{-3}$ & 1.315 \\
\hline Vol-lim-19.5 & -19.5 & 0.089 & 261.3 & 149773 & 22524 & 4.433 & 0.480 & 0.108 & 53.8 & $1.73 \times 10^{-3}$ & 1.504 \\
\hline Vol-lim-20.0 & -20.0 & 0.110 & 322.6 & 163094 & 24258 & 5.321 & 0.515 & 0.097 & 47.9 & $9.88 \times 10^{-4}$ & 1.856 \\
\hline Vol-lim-20.5 & -20.5 & 0.136 & 397.2 & 164004 & 23007 & 6.541 & 0.548 & 0.084 & 39.9 & $5.01 \times 10^{-4}$ & 2.596 \\
\hline Vol-lim-21.0 & -21.0 & 0.168 & 486.2 & 125016 & 14155 & 8.766 & 0.577 & 0.066 & 28.1 & $1.68 \times 10^{-4}$ & 4.463 \\
\hline
\end{tabular}

Notes. ${ }^{(a)}$ Galactic-extinction-corrected Petrosian magnitude limit. Columns are as follows: (1) absolute magnitude limit for volume-limited samples. (2) Maximum redshift. (3) Maximum comoving distance. (4) Number of galaxies in a sample. (5) Number of groups in a sample. (6) Mean pairwise separation of galaxies in comoving coordinates. (7) Used linking length (LL) in physical coordinates. (8) LL value in units of mean pairwise separation: $b_{\mathrm{com}}=b_{\mathrm{gal}}(1+z)$. (9) Fraction of galaxies in groups. (10) Group number density. (11) Luminosity weight for volume-limited samples.

Figure 2 shows the absolute magnitude $M_{r}$ of the galaxies as a function of distance. We see that the faintest galaxies are missing farther away. The inner panel in Fig. 2 displays the corresponding amount of luminosity that is missing at each distance (see Appendix C for details).

We constructed seven volume-limited galaxy samples with different magnitude $\left(M_{r}\right)$ cuts: $-18.0,-18.5,-19.0,-19.5$, $-20.0,-20.5$, and -21.0 . Since we complemented the original SDSS dataset with redshift measurements from other surveys, no upper magnitude limit was applied. The corresponding samples are shown in Fig. 2 as solid blue lines. The number of galaxies in each sample and the completeness distance limits are given in Table 1. The upper distance limit for the flux-limited sample is shown with the dashed line in Fig. 2 and is calculated from Eq. (1) using the average $k$-correction near the survey limit.

The observed distances (redshifts) of galaxies are commonly used to calculate absolute luminosities and to construct volumelimited samples. Considering that groups and clusters are extended objects in the redshifts space (the so-called finger-ofgod effect, Tully \& Fisher 1978), we have used the flux-limited group mean distances here to construct the volume-limited samples (for galaxies in flux-limited groups we used the group mean redshift instead of the redshift of each galaxy). However, this choice affects only a small fraction of galaxies close to the magnitude limits. For the current dataset, there are fewer than 100 affected galaxies for each volume-limited sample. Because of the same effect, the volume-limited samples are also affected close to the upper distance limit. In this case, we also decided to use flux-limited group mean distances instead of galaxy redshifts. We note that regardless of the choice of distance measurement, the galaxy and constructed group samples are always incomplete close to the survey boundaries.

\section{Method}

\subsection{Flux-limited galaxy groups/clusters}

We followed the basic steps of the method described in Tago et al. $(2008,2010)$ to extract groups from the flux-limited galaxy sample. Below, we briefly outline the method and report on the improvements made with respect to Tago et al. $(2008,2010)$.

Our group-finding is based on the friends-of-friends (FoF) algorithm (Turner \& Gott 1976; Press \& Davis 1982). This method links galaxies to systems using a certain neighbourhood radius, the linking length (LL). For each galaxy, all neighbours within the LL radius are considered to belong to the same system. The number and richness of the detected groups strongly depend on the chosen LL. In most cases, LL is not taken to be constant, but is allowed to vary with distance and/or other parameters.

Our experience shows that the choice of the LL depends on the goals of the specific study. Here, our aim is to find as many groups as possible, while keeping the general group properties uniform with respect to distance. In our group definition, we have tried to avoid including large sections of the surrounding large-scale filaments and parts of superclusters.

The applied FoF group construction method has been tested on mock galaxy surveys in Nurmi et al. (2013) and Old et al. (2014). Both papers indicate that the used FoF parameters (LL in radial and transversal direction) provide statistically reliable groups. In addition, the FoF parameters used here agree well with the values applied by others (see Duarte \& Mamon 2014).

To find the proper scaling of LL with distance, we firstly calculated the mean distance (in physical coordinates) to the nearest galaxy in the plane of the sky. The neighbour was sought within a cylindrical volume: the ratio of the radial to the transversal LLs was taken to be 10 (after transforming the radial LL in units of $\mathrm{km} \mathrm{s}^{-1}$ into a formal distance in $h^{-1} \mathrm{Mpc}$ ). The smallest cylinder that contains two galaxies defines the minimum distance between the two galaxies, and thus also the scaling for the LL. The cylinder diameter as a function of distance is shown in Fig. 3 with the green shaded area, the dotted green line shows the running mean of the distances. Below $z=0.1$, the nearest neighbour galaxy usually belongs to the same group. Farther away, the nearest galaxy typically belongs to another group, therefore the distance between neighbouring galaxies is increasing rapidly. This increase of the mean distance is an expected effect of a fluxlimited survey. A similar LL determination method was used by Old et al. (2014), where various group-finding algorithms (including the one presented here) are compared. Old et al. (2014) showed that the groups extracted using such LL strategy are statistically correct and the corresponding group properties are meaningful.

To construct our flux-limited group sample, we made an initial guess for the LL. For that, an arctan law

$d_{\mathrm{LL}}(z)=d_{\mathrm{LL}, 0}\left[1+a \arctan \left(z / z_{\star}\right)\right]$

was fitted to the scaling relation of the nearby galaxy sample $(z<0.1)$. In Eq. (2) $d_{\mathrm{LL}, 0}$ is LL at $z=0$, and $a$ and $z_{\star}$ are free 
parameters. Using the resultant LL law, we created a test group catalogue. Next we selected all the groups within the nearby volume $\left(d<200 h^{-1} \mathrm{Mpc}\right)$ with 15 to 50 members (altogether 539 groups). Smaller groups were excluded to reduce noise, and the largest groups were expelled to avoid large nearby clusters. The remaining richness range represents the groups in the nearby Universe well. Naturally, the scaling relation depends on the used group-richness bin, with smaller groups yielding lower LL values, and vice versa. The range of 15-50 members was chosen here to roughly match the mean distance to the nearest galaxy in the plane of the sky, e.g. to match the initial scaling relation.

Our next task was to find similar groups at distances where only the brightest members are seen. Assuming that the group members are all at the mean distance of the group, we determined their absolute magnitudes and peculiar radial velocities. Then we re-calculated the parameters of the groups as they would appear if the groups were located at larger distances. As more and more fainter group members fall outside the observable apparent magnitude limit with increasing distance, the group membership changes. We calculated the minimum FoF LL necessary to keep the group together at each distance. Since by definition groups are gravitationally bound systems, we kept the physical size of the groups while shifting them to greater distances. The mean required LL value (in physical coordinates) is shown in Fig. 3 with the dashed red line. The figure shows that to keep the group-finding consistent, the LL has to be increased moderately with distance as in our previous papers (Tago et al. 2008, 2010; Tempel et al. 2012). We fitted an arctan law to the new scaling relation and created a new test group catalogue and repeated the procedure. After a few iterations, the relation converged at the following parameters: LL at a redshift $z=0$ is $0.23 h^{-1} \mathrm{Mpc}$ and the parameters are $a=1.4$ and $z_{\star}=0.09$. The final law is shown in Fig. 3, where we also show the scaling relation as found in Tago et al. (2010).

Since galaxy locations in the radial direction are drawn from the redshift space, galaxy groups appear to be elongated along the radial direction. We have taken the ratio of the radial LL (in $h^{-1} \mathrm{Mpc}$ ) to the transversal LL (in $\mathrm{km} \mathrm{s}^{-1}$ ) to be 10 , yielding an initial radial LL value $230 \mathrm{~km} \mathrm{~s}^{-1}$. The value 10 corresponds to the average elongation of the groups along the radial direction (see Fig. 11). The resulting shape of the LL distribution is cylindrical. One may argue whether it might be better to use an ellipsoidal LL distribution. Tests have shown, however, that the cylindrical kernel reproduces recovered galaxy groups better (Eke et al. 2004).

As shown in Tago et al. (2008, 2010), the given LL determination leads to reasonable group properties. Our final group catalogues are very homogeneous. The richness, mean size, and velocity dispersion of a group are practically independent of the group distance (see below). The homogeneity of our catalogues has also been tested by other authors. For example, Tovmassian $\&$ Plionis (2009) have selected poor groups from our previous SDSS catalogues and concluded that the main parameters of our groups are distance-independent and well suited for statistical analysis.

Our final group catalogue contains 82458 groups with two or more members. Almost half of the galaxies in the sample $(48 \%)$ belong to a group.

We note that the choice of the LL in transversal and radial direction is rather arbitrary, and we have chosen the values based on our previous experiences. In several papers (e.g. Eke et al. 2004; Berlind et al. 2006; Yang et al. 2007; Robotham et al. 2011) the mock catalogues were used to find the "best" values

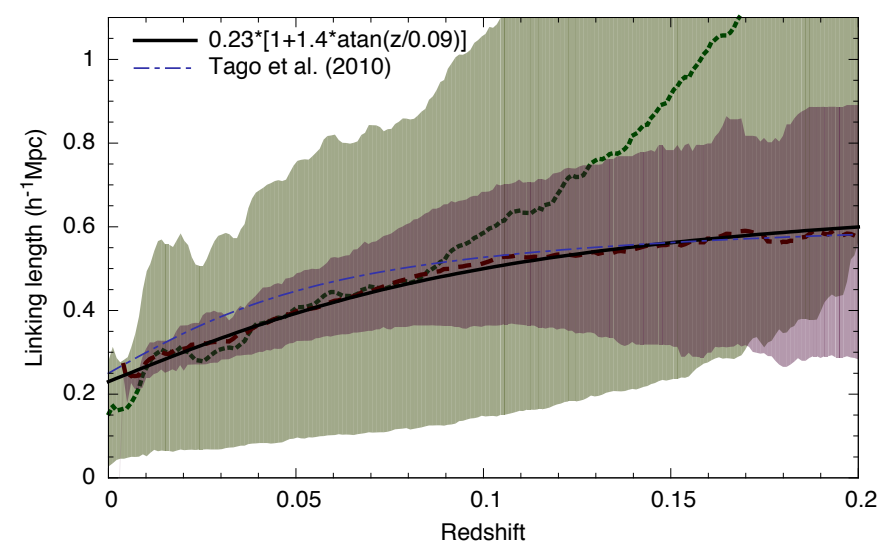

Fig. 3. FoF LL (in physical coordinates) as a function of redshift. The dotted green line shows the mean distance to the nearest neighbouring galaxy in physical coordinates with $68 \%$ confidence limits (green region). The dashed red line shows the LL scaling derived after shifting the nearby groups to larger distances, together with $68 \%$ confidence limits (red region). The solid black line shows the best fit to the scaling relation. The dot-dashed blue line shows the scaling from Tago et al. (2010).

for FoF parameters. However, no universally good value exists. Duarte \& Mamon (2014) analysed the effect of LL on the detected groups and concluded that the choice of the LL determines the fragmentation, merging, completeness, and reliability of the constructed group catalogue. They also showed that the LL values used here are close to the average of the values applied by others. Therefore, according the current knowledge, the group-finding algorithm presented in this paper is well founded.

\subsection{Construction of volume-limited groups/clusters}

Volume-limited groups are based on volume-limited galaxy samples, as described in Sect. 2.3. Since the galaxy number density in volume-limited samples is constant by definition, we used a constant LL (in physical coordinates) for each volume-limited sample. The LL was chosen to produce groups that are statistically similar to those found for a flux-limited sample. To achieve this, the LL was chosen based on the LL scaling found for the flux-limited sample. For each volume-limited sample, the LL was scaled according to the upper distance limit of the given volume-limited sample. At this distance, the number density of galaxies in the flux-limited sample is about the same as in the volume-limited sample. The LL values are given in Table 1 and are shown in Fig. 4 as solid black lines.

It is important to note that we intended to detect (mostly) virialised groups. We therefore considered the LL value in the physical instead of the comoving coordinates, expecting the groups to resist the cosmological expansion. In our previous volume-limited catalogues (Tago et al. 2010), comoving coordinates were used. However, the difference between the two choices is relatively small within the given redshift range, so it does not affect the general properties of the produced groups.

In general, there is no strict rule for choosing the LL for the FoF algorithm, and the LL is slightly different in various papers. For example, Berlind et al. (2006) used the value $b_{\text {com }}=0.14$ and the ratio of the radial-to-transversal LL was taken to be 5.4. The value $b_{\text {com }}$ is given in units of the mean pairwise separation of galaxies in the survey. Eke et al. (2004) adopted the values $b_{\text {com }}=0.13$ and the ratio of the radial-to-transversal LLs was taken to be 11 . The value $\left(b_{\text {com }}=b_{\text {gal }}(1+z)\right)$ in our catalogue 

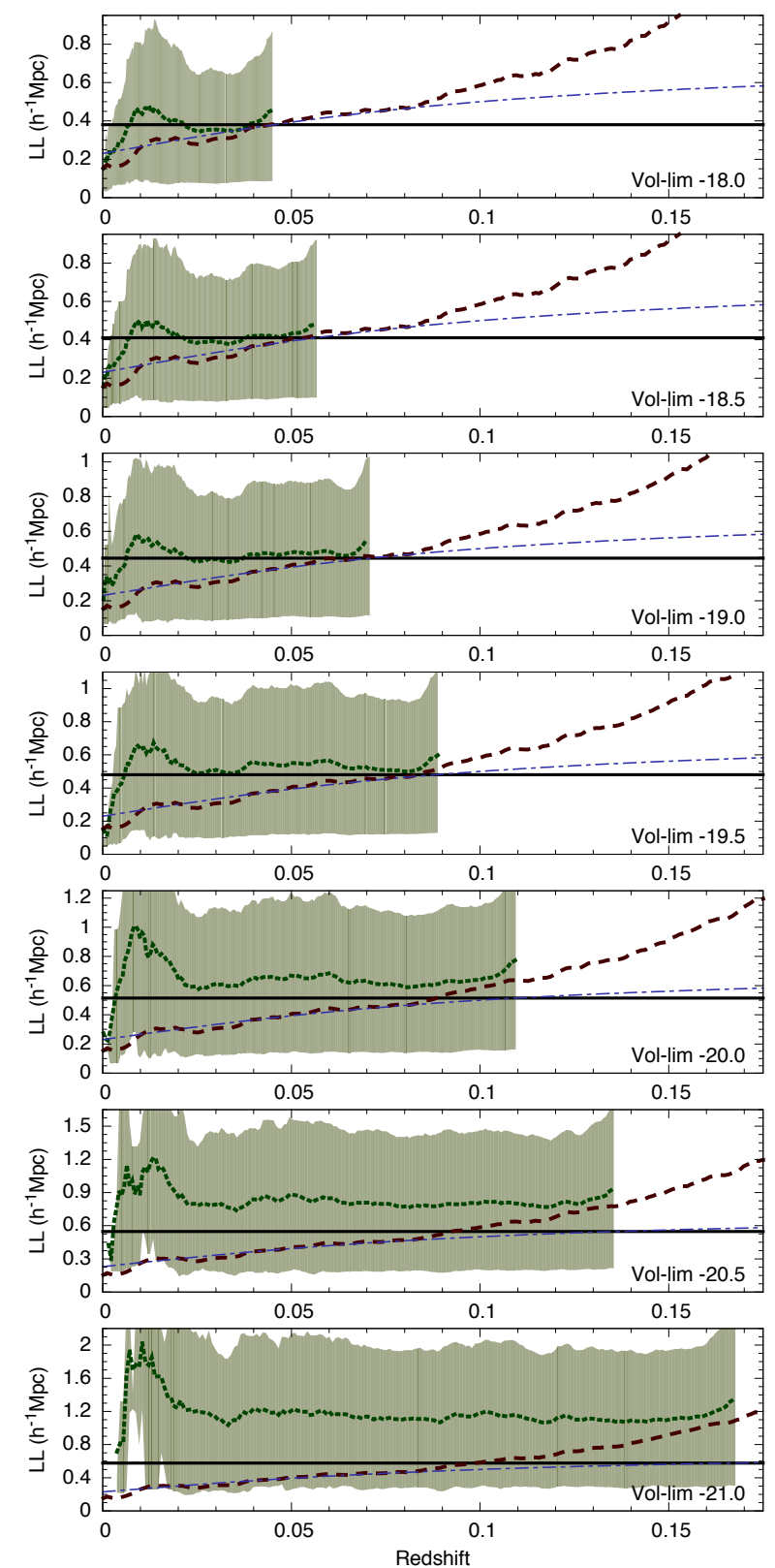

Fig. 4. Mean distance to the nearest galaxy (in physical coordinates) in various volume-limited samples together with $68 \%$ confidence limits (dotted green lines with shaded areas) as a function of redshift. For comparison, the mean distance to the nearest galaxy in the flux-limited sample is shown (dashed red lines). Dash-dotted blue lines show the LL scaling relation as found for the flux-limited sample, and solid black lines show the LL we used for volume-limited samples (in physical coordinates).

is redshift dependent (because of the constant LL in physical units) and is slightly lower (up to 0.12) than that used by Eke et al. (2004) and Berlind et al. (2006). The ratio of the radial-totransversal LLs in our catalogues is slightly lower than in Eke et al. (2004) and higher than in Berlind et al. (2006). Compared with our previous volume-limited catalogues based on SDSS DR7 (Tago et al. 2010), the LL values used in this paper are higher by up to $50 \%$, depending on the volume-limited sample and distance.

Recently, Duarte \& Mamon (2014) performed an in-depth study for the choice of the LL in FoF algorithms. They concluded that the optimal LL depends on the scientific goal for

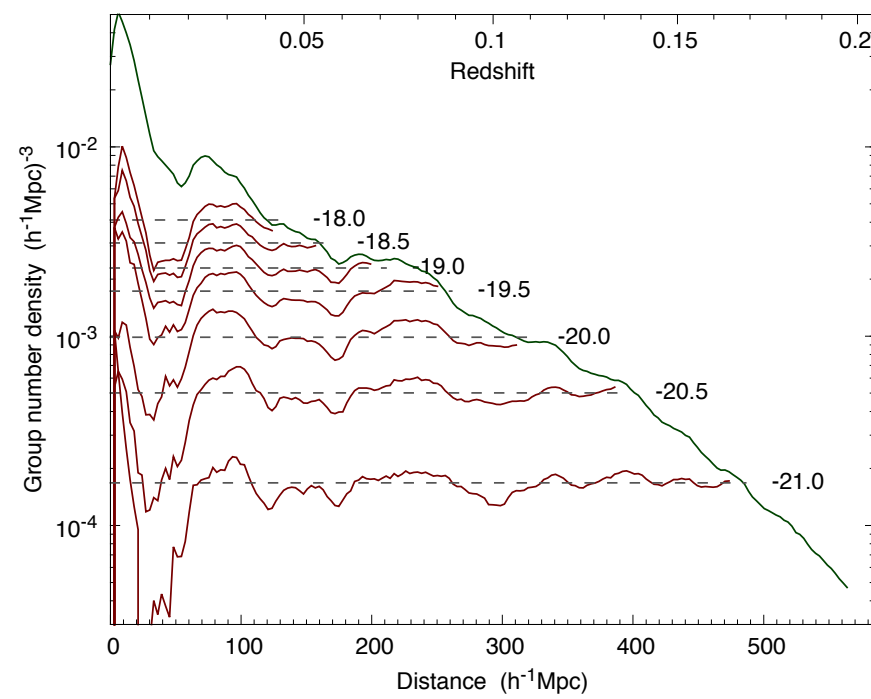

Fig. 5. Group number density as a function of distance for flux- and volume-limited samples. The green line shows the flux-limited sample, red lines show different volume-limited samples. Dashed lines give the average number density for the volume-limited samples (the values are given in Table 1). The density minimum at lower distances is caused by the low statistics and a small volume.

the group catalogue. The LL values used in our catalogue are within the range proposed by Duarte \& Mamon (2014).

Figure 4 shows the mean distance to the nearest galaxy (in physical coordinates) in the volume-limited samples as a function of distance (green dotted lines). For comparison, the mean distance in the flux-limited sample is shown (dashed red lines). Figure 4 shows that in the volume-limited samples, the mean distance to the nearest galaxy is almost independent of redshift, as expected. The deviations in the nearby region $(z<0.02)$ are caused by the small sample volume. The slight increase close to the upper distance limit is due to the limit itself.

For comparison, Fig. 4 shows the LL scaling relation for the flux-limited sample (dot-dashed blue lines) and the LL in the volume-limited samples (solid black lines). We note that for the four fainter volume-limited samples, the LL is about the same as the mean distance to nearest galaxy. For the brighter volume-limited samples, the mean distance to the nearest galaxy is larger than the LL. This is because the LL scaling relation in the flux-limited sample deviates from the mean distance to the nearest galaxy for redshifts $z>0.1$. Since our aim was to construct volume-limited group catalogues that are comparable with the flux-limited catalogue, the LL for the volumelimited samples was taken from the scaling relation found for the flux-limited sample, rather than found independently for each volume-limited sample. This choice provided volume-limited groups that are well comparable with the flux-limited groups (see Sect. 5.2).

Figure 5 shows the number density of groups for the fluxlimited sample and for various volume-limited samples. The number density of groups in the flux-limited sample decreases. For the volume-limited samples it is almost constant, as expected. The only differences are seen in the nearby regions, where the cosmic variance due to the small volume has a large effect. This figure shows that the groups extracted from the volume-limited samples are statistically homogeneous across the given volumes. Note that Fig. 5 does not characterise the groupfinding method itself. The deviations seen in the distributions are caused by the large-scale structure and the limited sample sizes. 


\subsection{Group and galaxy properties}

In this section, we describe the galaxy and group parameters that are included in the catalogues. These were also given in our previous catalogue (Tempel et al. 2012).

For each galaxy, we have estimated the galaxy morphology as described in Tempel et al. (2011). In Tempel et al. (2012) the morphologies were compared with those provided by Huertas-Company et al. (2011), and a very good agreement was found. In the present catalogue, galaxies are classified as spirals or ellipticals if the morphology in both catalogues agree. Otherwise, the morphology is marked as unclear.

The velocity dispersion $\sigma_{v}^{2}$ for groups were calculated with the standard formula

$\sigma_{v}^{2}=\frac{1}{\left(1+z_{\mathrm{m}}\right)^{2}(n-1)} \sum_{i=1}^{n}\left(v_{i}-v_{\text {mean }}\right)^{2}$,

where $v_{\text {mean }}$ and $z_{\mathrm{m}}$ are the mean group velocity and redshift, $v_{i}$ is the velocity of an individual group member, and $n$ is the number of galaxies with observed velocities within the group.

The extent of the group in the plane of the sky is defined as

$\sigma_{\text {sky }}^{2}=\frac{1}{2 n\left(1+z_{\mathrm{m}}\right)^{2}} \sum_{i=1}^{n}\left(r_{i}\right)^{2}$,

where $r_{i}$ is the projected distance in the sky from the group centre (in comoving coordinates, in units of $h^{-1} \mathrm{Mpc}$ ), and $z_{\mathrm{m}}$ is the mean redshift of the group.

The virial radii $R_{\text {vir }}$ of the groups are calculated from the formula

$\frac{1}{R_{\mathrm{vir}}}=\frac{2}{\left(1+z_{\mathrm{m}}\right) n(n-1)} \sum_{i \neq j}^{n} \frac{1}{R_{i j}}$,

where $R_{i j}$ is the projected distance between galaxies in pairs in a group.

Figure 6 shows the luminosity function in the $r$-filter for our final sample of the SDSS galaxies. It was calculated using an adaptive smoothing kernel as described in Tempel et al. (2011). The vertical lines mark the limits for the different volumelimited samples described above. For comparison, we show the best-fit analytical luminosity functions from previous data releases: Tempel et al. (2012, DR8), Montero-Dorta \& Prada (2009, DR6), and Blanton et al. (2003, EDR).

We used the double power-law to find an analytical approximation of the luminosity function (see Fig. 6),

$n(L) \mathrm{d} L \propto\left(L / L^{*}\right)^{\alpha}\left[1+\left(L / L^{*}\right)^{\gamma}\right]^{\frac{\delta-\alpha}{\gamma}} \mathrm{d}\left(L / L^{*}\right)$,

where $\alpha$ is the exponent at lower luminosities $\left(L / L^{*}\right) \ll 1, \delta$ is the exponent at higher luminosities $\left(L / L^{*}\right) \gg 1, \gamma$ is a parameter that determines the speed of transition between the two power laws, and $L^{*}$ is the characteristic luminosity of the transition. We find the best match with the observed luminosity function with $\alpha=-1.250 \pm 0.008, \delta=-7.32 \pm 0.30, \gamma=1.71 \pm 0.05$, and $M^{*}=-21.88 \pm 0.06$ (corresponds to $L^{*}$ ).

Additionally, the catalogues include environmental densities of the galaxies and groups. These densities are important when analysing the influence of the local and/or global environments on galaxy evolution. Local and global densities are estimated using different smoothing radii. The density calculation is described in Appendix C.

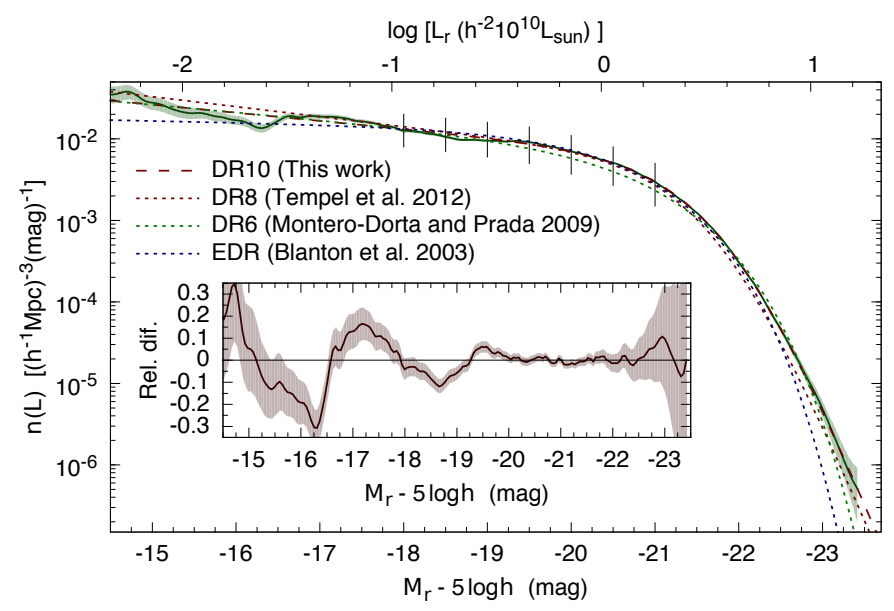

Fig. 6. Galaxy luminosity function in the $r$-filter. The solid line and the shaded region show the measured luminosity function together with the $99.7 \%$ confidence limit (corresponds to $3 \sigma$ ). The dashed line shows the best-fit double power-law fit. Dotted lines show previous approximations by Blanton et al. (2003, EDR), Montero-Dorta \& Prada (2009, DR6), and Tempel et al. (2012, DR8). The former two are approximations with Schechter functions. The inset panel shows the relative difference between the best-fit double power-law function and the measured luminosity function. Vertical lines show the magnitude limits for the volume-limited samples.

\section{Dynamical masses of the groups and clusters}

To estimate the total masses of galaxy systems in the catalogue, we used the virial theorem $2 T=U$, where $T$ is kinetic energy and $U$ is potential energy. Expressing

$T=\frac{M_{\mathrm{tot}} \sigma_{v}^{2}}{2}, \quad U=G \frac{M_{\mathrm{tot}}^{2}}{R_{\mathrm{g}}}$,

where $\sigma_{v}$ is the velocity dispersion within the system, $M_{\mathrm{tot}}$ is the total mass of the system, $G$ is the gravitational constant, and $R_{\mathrm{g}}$ is the gravitational radius, we can derive the following relation for the total mass:

$M_{\mathrm{tot}}=2.325 \times 10^{12} \frac{R_{\mathrm{g}}}{\mathrm{Mpc}}\left(\frac{\sigma_{v}}{100 \mathrm{~km} \mathrm{~s}^{-1}}\right)^{2} M_{\odot}$.

This means that to estimate the masses of our FoF groups, we have to find two quantities: $R_{\mathrm{g}}$ and $\sigma_{v}$. The velocity dispersion was estimated using the line-of-sight velocities of all detected galaxies of a galaxy system. The calculated quantity is the onedimensional velocity dispersion $\sigma_{v 1 \mathrm{D}}$ (Eq. (3)). Assuming dynamical symmetry, the real (3D) velocity dispersion in groups would thus be $\sigma_{v}=\sqrt{3} \sigma_{v 1 \mathrm{D}}$.

According to Binney \& Tremaine (2008), $R_{\mathrm{g}}$ can be found by equalising the potential energy (7) with the equation

$U=4 \pi G \int_{0}^{R_{\text {out }}} \frac{M(r)}{r} \rho(r) r^{2} \mathrm{~d} r$,

where $M(r)$ is the mass within a sphere $r$ and $R_{\text {out }}$ is the outer limit of the system. The assumed density profile for the galaxy systems (groups/clusters) is $\rho(r)$.

To estimate $R_{\mathrm{g}}$ from observations, we used the observed dispersion in the plane of the sky $\sigma_{\text {sky }}$ (Eq. (4)). To do that, we assumed some density profile (NFW and Hernquist) and calculated the relation $\kappa=R_{\mathrm{g}} / \sigma_{\text {sky }}$ based on this density profile. We 
used $\kappa$ to transfer the observed $\sigma_{\text {sky }}$ to $R_{\mathrm{g}}$, which was then used in Eq. (8) to calculate the dynamical masses of the groups.

The derivation of the group masses for the cases of NFW and Hernquist mass distribution are described below. The masses are estimated only for groups with three or more members. For galaxy pairs, the group extent in the sky and velocity dispersion are not clearly defined. But for other poorer groups the estimated group mass is largely uncertain as well.

\subsection{NFW profile}

Assuming the NFW profile (Navarro et al. 1997), the $\rho(r)$ depends only on one parameter: the halo mass $M_{200}$, which all the other parameters depend on. The profile is expressed as

$\rho(r)=\frac{\delta_{c} \rho_{\text {crit }}}{\left(\frac{r}{R_{\mathrm{s}}}\right)\left(1+\frac{r}{R_{\mathrm{s}}}\right)^{2}}$,

where $\rho_{\text {crit }}$ is the critical density of the Universe and $R_{\mathrm{S}}$ is a scale radius. Defining the concentration as $c_{200}=R_{200} / R_{\mathrm{s}}$, where $R_{200}$ is the radius containing the mass $M_{200}$, while the mean density inside that radius is 200 times the critical density of the Universe, the $\delta_{c}$ is expressed as

$\delta_{c}=\frac{200}{3} \frac{c_{200}^{3}}{\ln \left(1+c_{200}\right)-\frac{c_{200}}{1+c_{200}}}$.

Following Macciò et al. (2008), the parameter $c_{200}$ is related to the halo mass $M_{200}$ as

$\log \left(c_{200}\right)=0.83-0.098 \log \left[\frac{M_{200}}{10^{12} h^{-1} M_{\odot}}\right]$.

To calculate the ratio $\kappa$ assuming the NFW profile, we took $R_{\text {out }}$ in Eq. (9) to be equal to $R_{200}$. This allowed us to calculate the quantity $\kappa$ for a fixed mass $M_{\text {tot }}$.

The $\sigma_{\text {sky }}$ for the NFW profile was calculated by integrating the projected NFW density (Bartelmann 1996; Łokas \& Mamon 2001).

The total mass of the group was found iteratively. Firstly, we assumed some mass $M_{\text {tot }}$, which was taken to be $M_{200}$ for the NFW profile. Using this $M_{200}$, we determined the NFW profile and computed $\kappa$ as described above. Using the estimated $\kappa$ value, we recalculated the total mass of group $M_{\text {tot }}$. Based on this mass, we recalculated $\kappa$. We iterated the process until it converged. This usually took fewer than ten iterations.

\subsection{Hernquist profile}

The Hernquist profile (Hernquist 1990) is similar to the NFW profile at the centre, but has a finite mass:

$\rho(r)=\frac{M}{2 \pi} \frac{a}{r} \frac{1}{(r+a)^{3}}$,

where $M$ is the total mass of the halo and $a$ is the scale radius.

For this profile, the total potential energy $U$ is

$U=-\frac{G M^{2}}{6 a}$

(see Eq. (14) in Hernquist 1990). This gives $R_{\mathrm{g}}=6 a$.

The scale factor $a$ is easy to find using the half-mass radius $R_{\mathrm{e}}$ of the projected density distribution, $R_{\mathrm{e}}=1.8153 a$ (see Eq. (38) in Hernquist 1990).

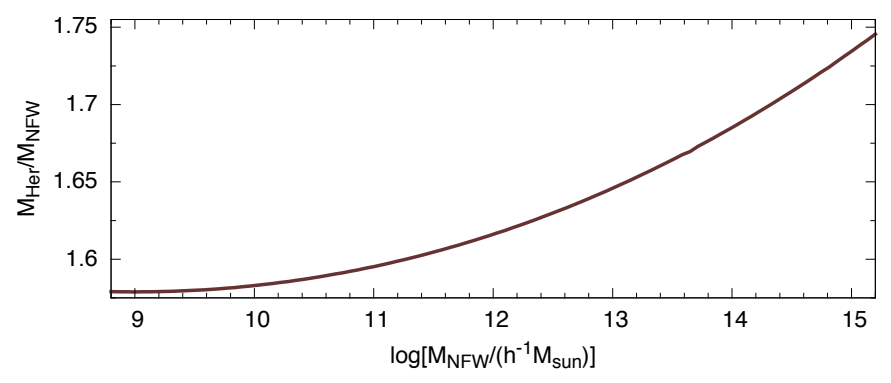

Fig. 7. Ratio of group dynamical masses calculated assuming Hernquist and NFW profiles as a function of group mass.

The half-mass radius $R_{\mathrm{e}}$ is the median of the sample of the projected galaxy distances from the centre of the group and can be estimated directly from observations. This estimate is noisy, especially for poor groups, and so we estimated it on the basis of our $\sigma_{\text {sky }}$ (Eq. (4)). This amounts to approximating the group galaxy distribution in the sky by a 2D Gaussian

$f(x, y) \mathrm{d} x \mathrm{~d} y=\frac{1}{2 \pi \sigma_{\text {sky }}^{2}} \exp \left(-\frac{x^{2}+y^{2}}{2 \sigma_{\text {sky }}^{2}}\right) \mathrm{d} x \mathrm{~d} y$,

where $x, y$ are any local Cartesian coordinates in the plane of the sky.

As $x^{2}+y^{2}=R^{2}$ and $\mathrm{d} x \mathrm{~d} y=2 \pi R \mathrm{~d} R$, this is the Rayleigh distribution for $R$ :

$f(R) \mathrm{d} R=\frac{1}{\sigma_{\text {sky }}^{2}} \exp \left(-R^{2} / 2 \sigma_{\text {sky }}^{2}\right) R \mathrm{~d} R$,

and

$\int_{0}^{\infty} R^{2} f(R) \mathrm{d} R=2 \sigma_{\mathrm{sky}}^{2}$

which yields the estimate

$\hat{\sigma}_{\text {sky }}^{2}=\frac{1}{2 N} \sum_{i=1}^{N} R_{i}^{2}$

that we give in our catalogue.

Now, for the Rayleigh distribution the integral probability $P\left(R<R_{a}\right)=F\left(R_{a}\right)=1-\exp \left(-R_{a}^{2} / 2 \sigma_{\text {sky }}^{2}\right)$. The half-mass condition says $F\left(R_{\mathrm{e}}\right)=1 / 2$, giving $R_{\mathrm{e}}=\sigma_{\text {sky }} \sqrt{2 \ln 2}=1.386 \sigma_{\text {sky }}$. Equating this with the Hernquist $R_{\mathrm{e}}$ above, we obtain $R_{\mathrm{e}}=$ $1.386 \sigma_{\text {sky }}=1.8153 a$, hence $a=0.764 \sigma_{\text {sky }}$ and $R_{\mathrm{g}}=6 a=$ $4.582 \sigma_{\text {sky. }}$.

The estimated masses using Hernquist and NFW profiles are tightly related. Figure 7 shows the ratio of these two mass estimates. The Hernquist masses are 1.55-1.75 more massive, depending on the system mass. This can be also considered as the systematic bias that comes from assuming a fixed mass profile.

\section{Results: group and cluster catalogues}

\subsection{Properties of flux-limited groups and clusters}

Various properties of the flux-limited groups are shown in Fig. 8 as a function of distance. The main observational properties of the groups, radial velocity dispersion and group extent in the sky, do not depend much on distance. A slight correlation with distance is expected since the derived group properties depend 

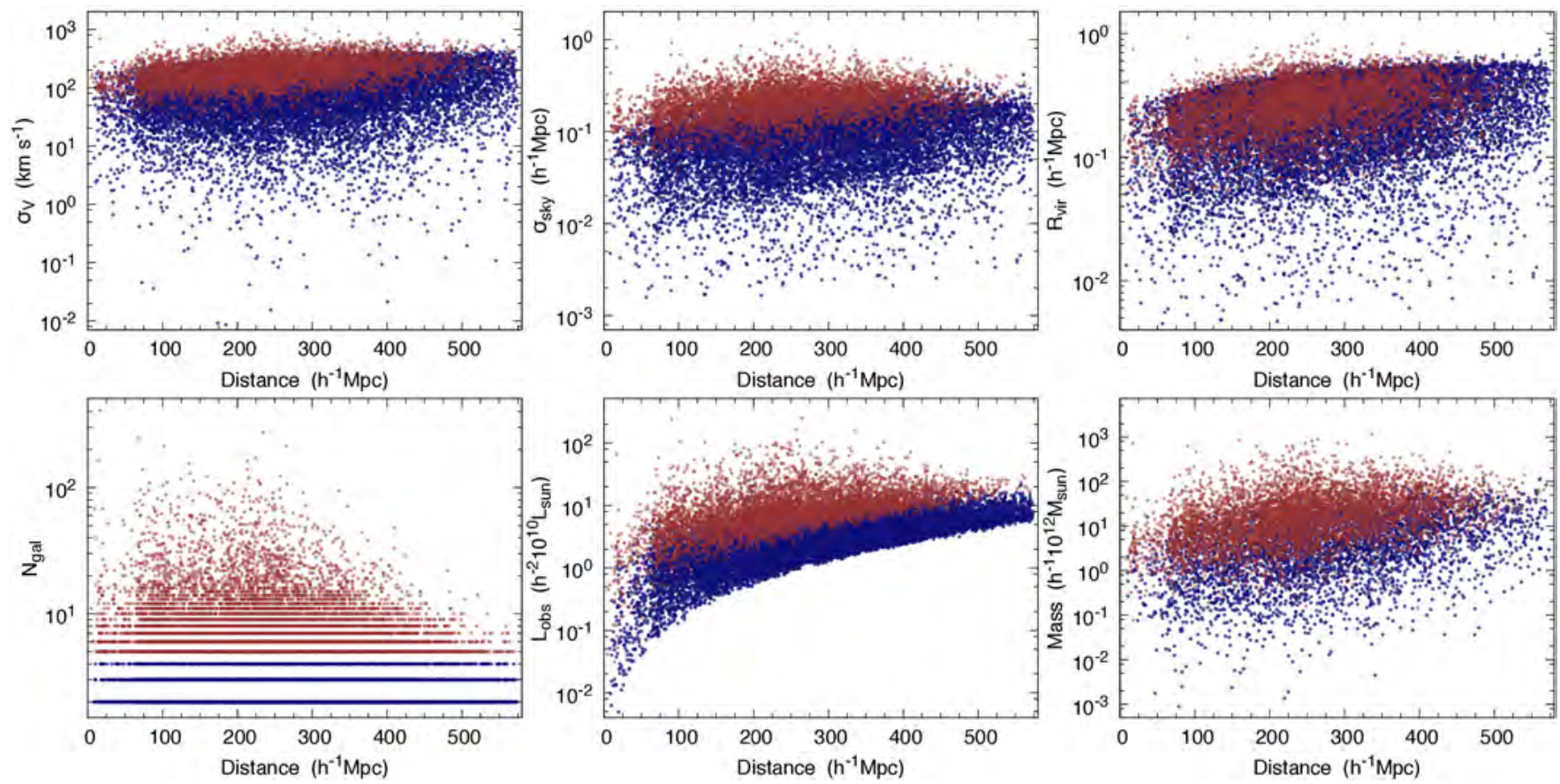

Fig. 8. Flux-limited group properties as a function of comoving distance. Upper left panel: velocity dispersion; upper middle: group extent in the plane of the sky; upper right: virial radius; lower left: number of galaxies in the group; lower middle: sum of the observed galaxy luminosities; lower right: mass of the galaxy group. Blue and red dots show groups with fewer than five and five or more members, respectively.
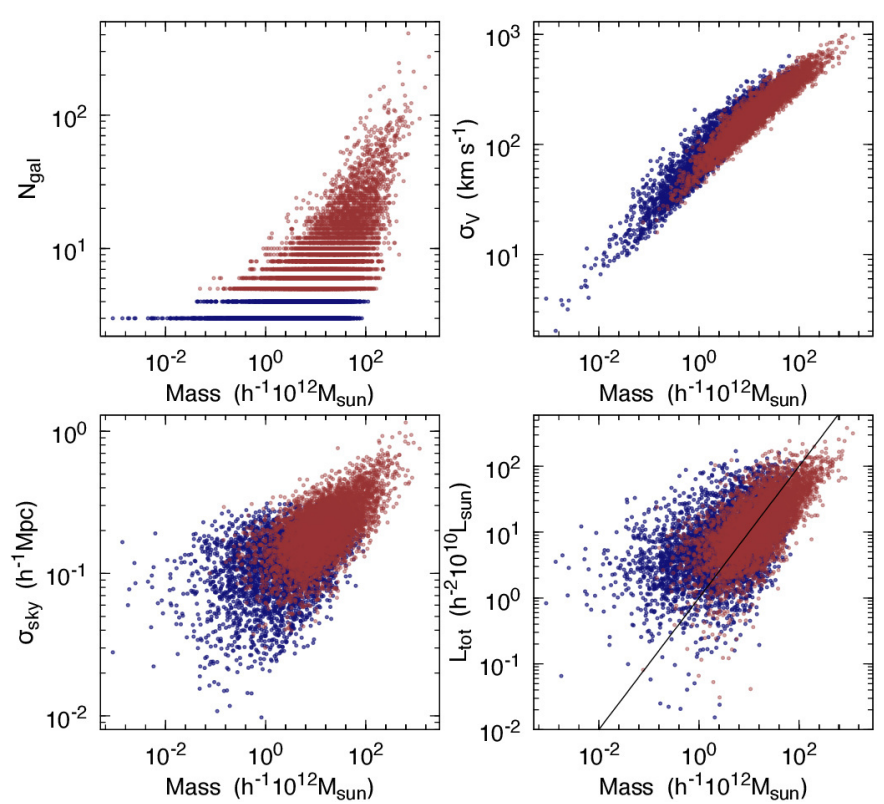

Fig. 9. Flux-limited group masses versus group properties: upper left panel: group richness; upper right: velocity dispersion; lower left: group extent; lower right: group total luminosity. Blue points denote small groups (fewer than 5 members) and red dots denote larger groups. Galaxy pairs are not shown.

on group richness and/or the imposed magnitude limit. For example, Old et al. (2013) showed that the velocity dispersion is slightly underestimated when only the brightest cluster members are considered. In general, Fig. 8 shows that the flux-limited selection effect has been largely eliminated when choosing the distance-dependent LL for the FoF.

The distribution of group richness as a function of distance (lower left panel in Fig. 8) indicates that farther away, richer groups become scarce. This is a natural result for a flux-limited survey. Nevertheless, some rich groups (more than 50 members) can be found up to $400 \mathrm{~h}^{-1} \mathrm{Mpc}$.

Figure 9 shows the flux-limited group richness, velocity dispersion, group extent, and the total luminosity of the group as a function of group mass. The tightest correlation is between the group mass and the velocity dispersion. This is expected since the virial theorem is most sensitive to velocity dispersion. The upper-left panel in this figure also shows that for small groups the scatter of mass estimate is very large. The scatter decreases while moving toward richer groups.

The relation between the group mass and the total luminosity of the group is plotted in the lower right panel in Fig. 9. The total luminosity given by Eq. (C.1) is compensated for by the luminosity of galaxies that are missed because of the magnitude limit of the survey. This estimate of the total luminosity is a statistically better characteriser of groups than the total observed luminosity or the group richness. However, it is not accurate for any particular group since the luminosity function depends on group properties (e.g. mass). The scatter in this plot is relatively large, but there is a correlation. Since the total luminosity can be used to estimate the environmental density (using the smoothed luminosity density fields), this plot indicates that the restored luminosity density field is a realistic characteriser of the environment.

Figure 10 indicates the group shape in the redshift space (the ratio of velocity dispersion to group extent in the plane of sky) as a function of the group mass, velocity dispersion, and group richness. The group shape does not depend on the group richness and there is a small dependency on the group mass. However, the group shape has a clear dependency on the group velocity dispersion. The groups/clusters with a high velocity dispersion are more stretched out in the redshift space. The same dependency was recently shown by Wojtak (2013).

In Fig. 10 the average elongation of groups is roughly 10 . The same value was used for the ratio between LLs in radial 

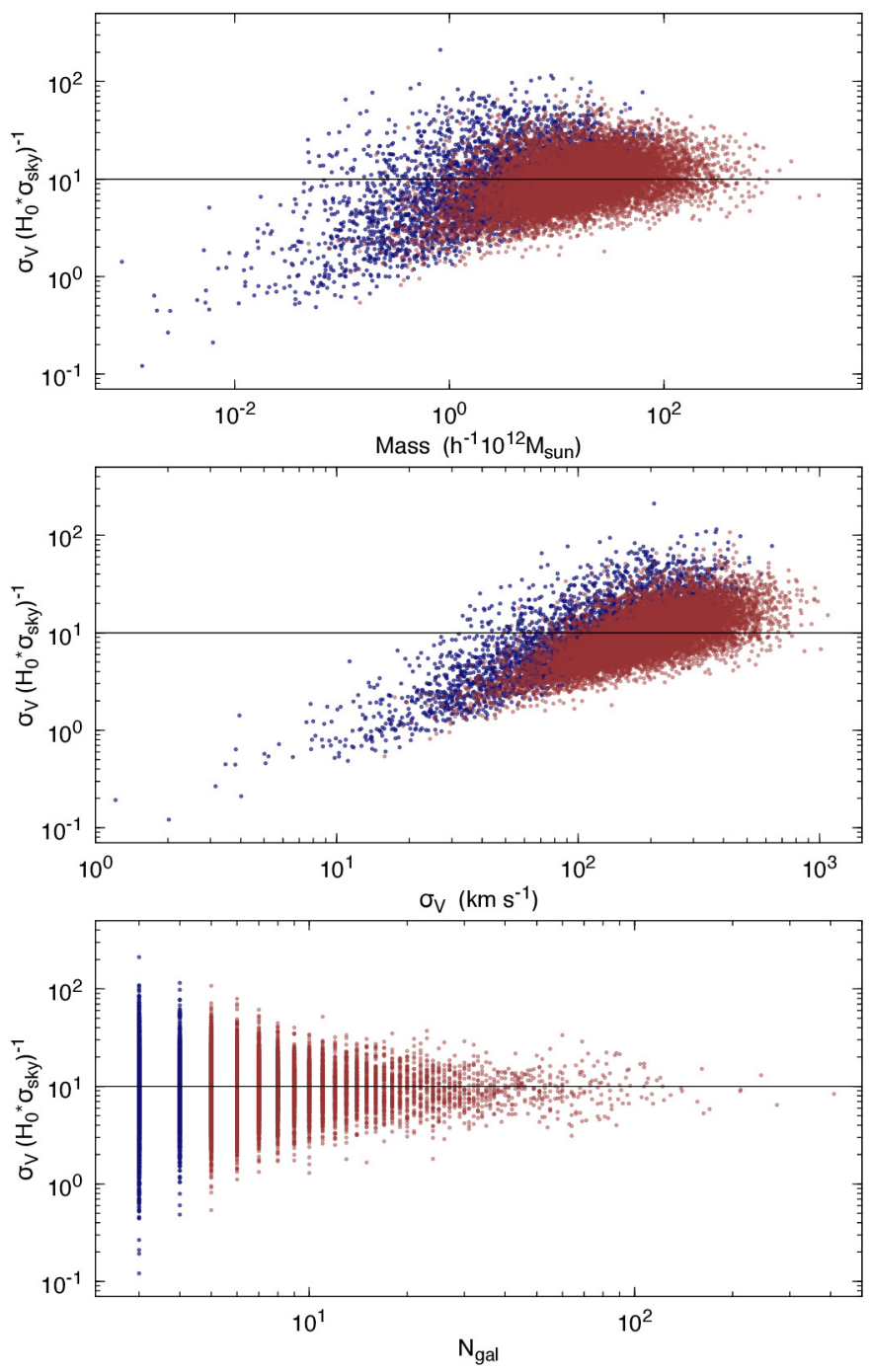

Fig. 10. Group shape in the redshift space (ratio of velocity dispersion to the group extent in the sky) as a function of group mass (upper panel), velocity dispersion (middle panel), and group richness (lower panel). Blue points show poor groups (fewer than five members) and red dots show richer groups.

and transversal directions. One might think that the derived average elongation of groups solely depends on the input LL ratios. To test this, we generated two test group catalogues with input LL ratios of 5 and 20, respectively, while all the other parameters were kept the same. The resulting average elongation of groups is shown in Fig. 11. The average elongation in each case is still roughly 10; only the smallest groups display a slight dependency on the input LL ratio. We conclude that the derived elongation of groups (except for poor groups) does not depend significantly on the input LL ratio.

\subsection{Volume-limited groups and mass function}

Table 2 lists the fraction of the volume-limited groups that are also present in the flux-limited sample. Matches between the volume- and flux-limited groups were found within a $1 h^{-1} \mathrm{Mpc}$. Overall, the percentage of matches is $80-90 \%$, which means that most of the volume-limited groups are identified at the same locations as the flux-limited groups. Table 2 also gives the fraction of groups in the volume-limited samples that have about the same mass as estimated for the corresponding groups in
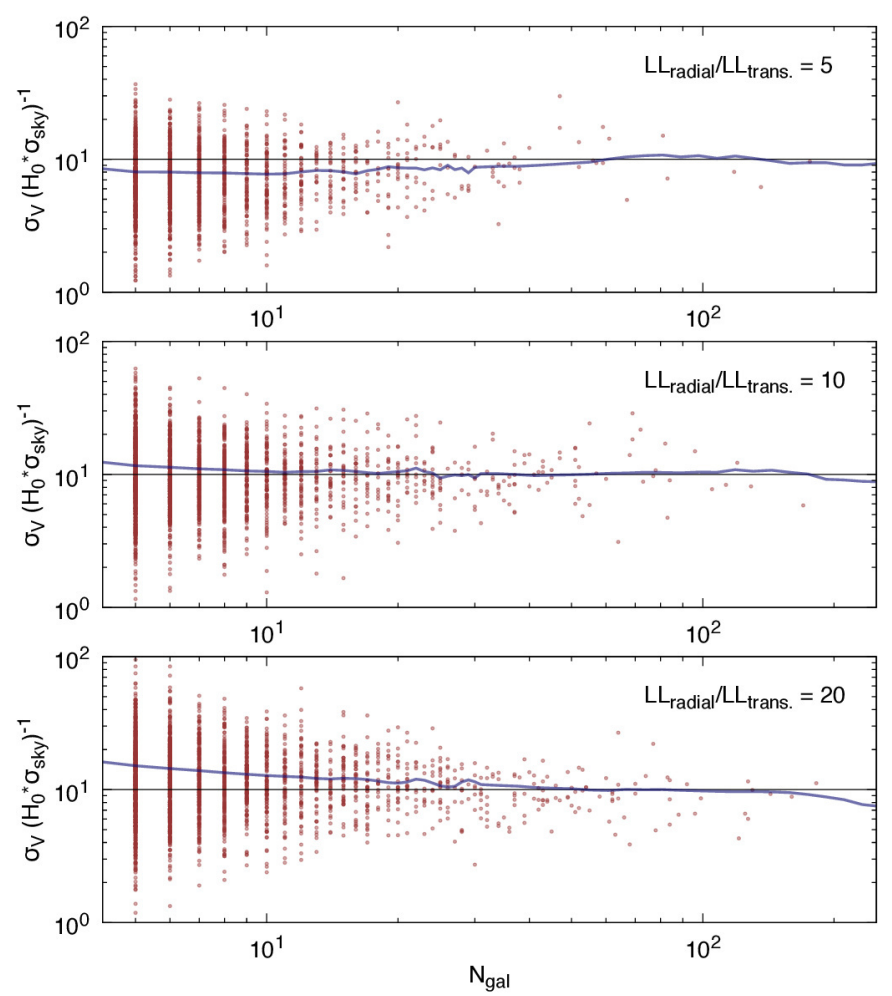

Fig. 11. Group shape in the redshift space as a function of group richness for different input LL ratios. The solid blue line shows the running mean for each sample.

Table 2. Volume-limited group statistics. Comparison with flux-limited groups.

\begin{tabular}{lccc}
\hline \hline Sample & $\begin{array}{c}\mathrm{Frac}^{a} \\
\%\end{array}$ & $\begin{array}{c}\text { Frac } 0.5^{b} \\
\%\end{array}$ & $\begin{array}{c}\text { Frac } 0.1^{c} \\
\%\end{array}$ \\
\hline Volume-limited-18.0 & 91 & 71 & 37 \\
Volume-limited-18.5 & 90 & 71 & 38 \\
Volume-limited-19.0 & 89 & 70 & 39 \\
Volume-limited-19.5 & 87 & 69 & 40 \\
Volume-limited-20.0 & 83 & 63 & 37 \\
Volume-limited-20.5 & 80 & 60 & 39 \\
Volume-limited-21.0 & 76 & 56 & 39 \\
\hline
\end{tabular}

Notes. ${ }^{(a)}$ Match fraction between volume- and flux-limited groups. The match radius is $1 h^{-1}$ Mpc. ${ }^{(b)}$ Fraction of matches where the relative mass difference is smaller than $0.5 .{ }^{(c)}$ Fraction of matches where the relative mass difference is smaller than 0.1 .

the flux-limited sample. Up to the relative mass difference 0.5 , the percentage of matches is $60-70 \%$. Even within the relative mass difference 0.1 , the percentage of matches is about $40 \%$. Considering the large scatter of the mass estimates (especially for poorer groups), the fact that majority of the groups has relatively similar mass estimates indicates the reliability of the mass determination method and also means that the groups identified using different samples are the same physical objects. We conclude that our strategy for the LLs in the volume-limited samples is appropriate and the volume-limited groups are well compatible with the flux-limited groups.

Figure 12 shows a comparison of the masses of the volumelimited groups and the masses of the corresponding flux-limited groups. For richer groups the masses in the flux- and volumelimited samples are very well correlated, indicating that the 

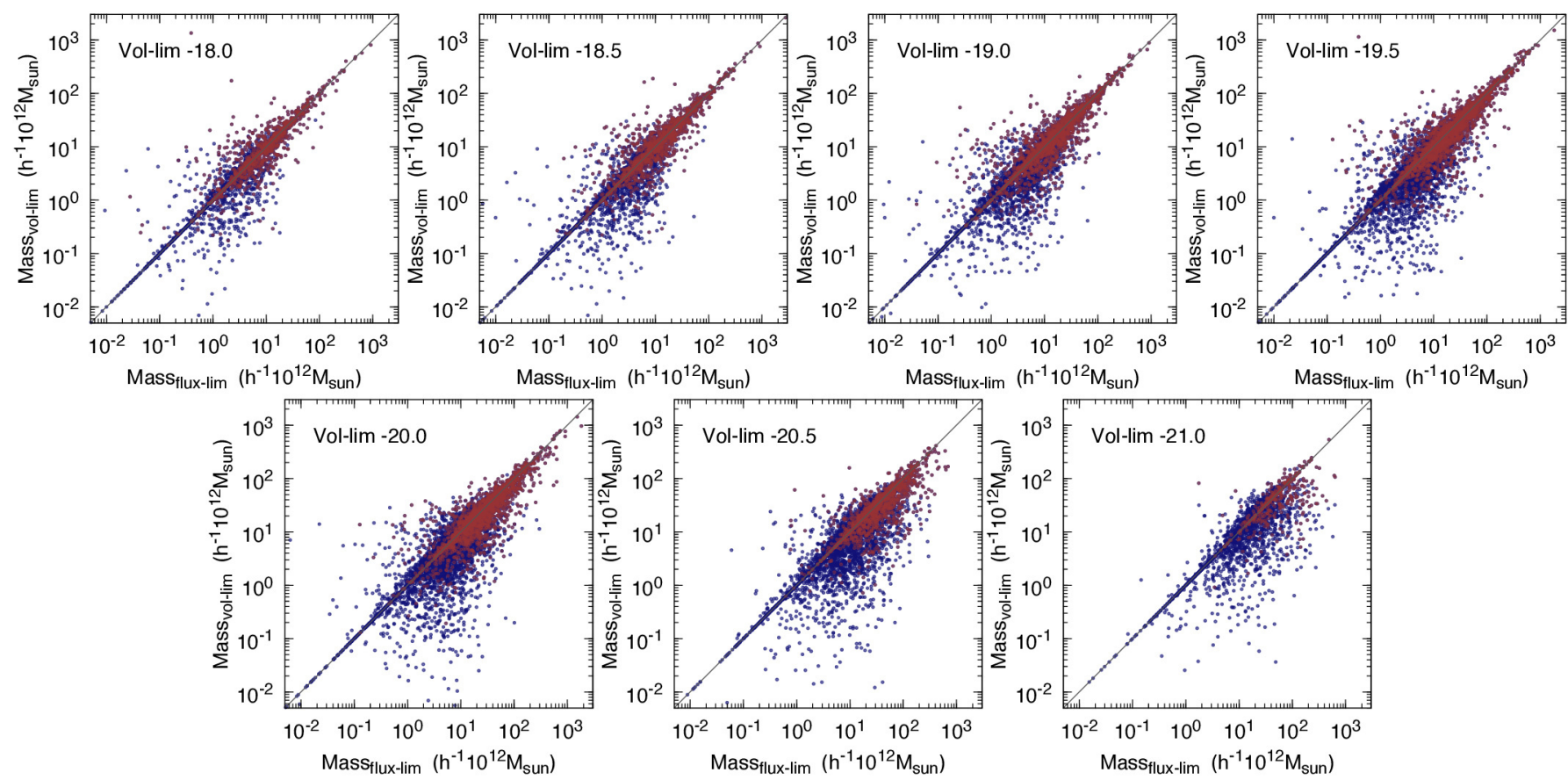

Fig. 12. Flux-limited group/cluster masses compared with the volume-limited group/cluster masses. Red points show the groups with five or more members and blue points show poorer groups. The solid grey line shows the one-to-one relationship between flux- and volume-limited masses.

groups from different samples are generally overlapping. For poorer groups, the masses in the volume-limited samples tend to be underestimated because too many group members are missed because of the flux limit.

The correspondence between the flux- and volume-limited samples can also be checked by comparing the resulting group mass functions, as shown in Fig. 13. The mass function for massive clusters in different samples is similar (except for the brightest volume-limited sample), thus the masses estimated using only the brightest cluster galaxies are as reliable as the masses estimated using also fainter galaxies. The mass function of groups in the brightest volume-limited sample with $M_{r, \lim }=$ -21.0 lies notably below the other samples. In this case, too few galaxies remain within the luminosity limits and the group sample is incomplete even for massive clusters. In several cases, only one to two brightest galaxies of each group/cluster remain within the luminosity limits and these groups/clusters are absent from Fig. 13. At lower masses, the samples start to progressively deviate from each other because the number of detected systems depends on the brightness limit.

For comparison, the cluster mass functions derived by Rines et al. (2007) are plotted in Fig. 13. They studied the cluster masses in an $X$-ray-selected sample of clusters with data from SDSS DR4. The masses were computed in two ways, one using the virial theorem (black squares) and the other (red squares) using the caustic technique (Rines \& Diaferio 2006). Both mass functions are similar to our mass functions for massive clusters. Rines et al. (2007) also compared their results with the mass functions estimated with other methods: using $X$-ray data (Reiprich \& Böhringer 2002) and using the mass-richness relation, applied on early SDSS data (Bahcall et al. 2003). All of these independent methods agree well in the massive-cluster domain.

Figure 13 shows that at the low end, the mass function depends on the applied magnitude limit. The higher the absolute magnitude limit, fewer groups and clusters are detected. The

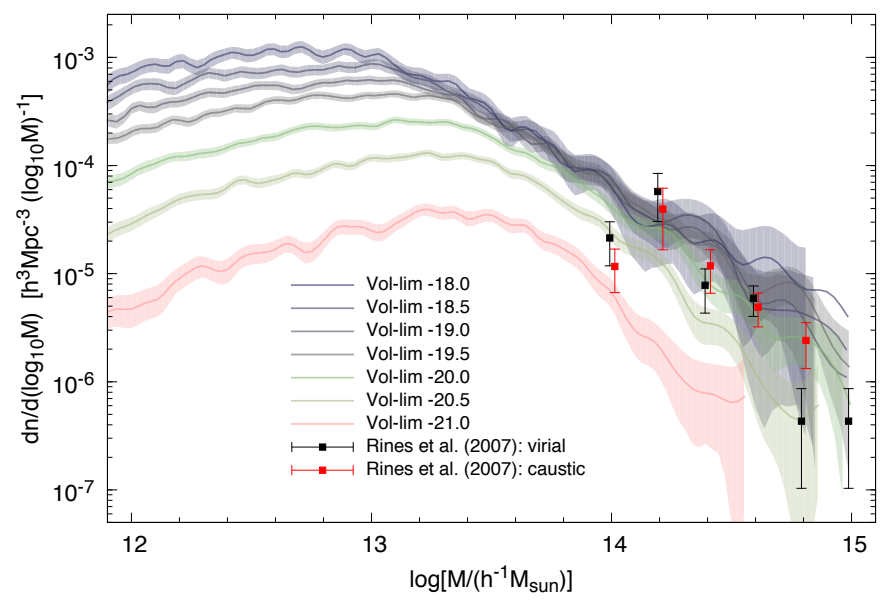

Fig. 13. Group mass function for various volume-limited samples (solid lines) together with $95 \%$ confidence limits (shaded regions). The mass function is calculated using groups with three or more members. Black and red points and error bars are the mass function from Rines et al. (2007) using virial masses and caustic masses, respectively (the error bars show $68 \%$ uncertainties).

slope of the mass function for different volume-limited samples is similar, which indicates that the group/cluster detection algorithm does not create mass-dependent systematic errors. In the near future, we plan to study the mass function of groups and clusters in the SDSS data and in different cosmological models in more detail.

\section{Conclusions}

We have updated and improved our previous galaxy group catalogues, which are constructed on the basis of the FoF method, following a similar procedure as used in previous papers (Tago et al. 2008, 2010; Tempel et al. 2012). The group-finding method 
was applied to flux- and volume-limited samples drawn from the SDSS main contiguous area, covering 7221 square degrees in the sky. In addition to the SDSS spectroscopic redshifts, the galaxy sample was complemented with redshifts from the 2MRS, 2dFGRS, and RC3 catalogues.

As an important addition, we estimated the group/cluster dynamical masses using the virial theorem. We showed that the groups extracted from the flux- and volume-limited samples are well compatible with each other. We calculated the mass functions for the volume-limited samples. At the massive end, the functions agree with previous observational estimates (Rines et al. 2007).

As a next step, we plan to use the new catalogue to map the large-scale galactic filaments (similarly to Tempel et al. 2014) and to construct a catalogue of superclusters (similarly to Liivamägi et al. 2012).

Acknowledgements. We thank our colleagues from Tartu Observatory and Tuorla Observatory for carefully checking the catalogues. We acknowledge support by the Estonian Research Council grants 9428, MJD272, PUT246, IUT26 and from the Centre of Excellence of Dark Matter in (Astro)particle Physics and Cosmology. All the figures have been made using the gnuplot plotting utility. This research has made use of the NASA's Astrophysics Data System Bibliographic Services. Funding for SDSS-III has been provided by the Alfred P. Sloan Foundation, the Participating Institutions, the National Science Foundation, and the US Department of Energy Office of Science. The SDSS-III web site is http://wWw.sdss3.org/. SDSS-III is managed by the Astrophysical Research Consortium for the Participating Institutions of the SDSS-III Collaboration including the University of Arizona, the Brazilian Participation Group, Brookhaven National Laboratory, Carnegie Mellon University, University of Florida, the French Participation Group, the German Participation Group, Harvard University, the Instituto de Astrofísica de Canarias, the Michigan State/Notre Dame/JINA Participation Group, Johns Hopkins University, Lawrence Berkeley National Laboratory, Max Planck Institute for Astrophysics, Max Planck Institute for Extraterrestrial Physics, New Mexico State University, New York University, Ohio State University, Pennsylvania State University, University of Portsmouth, Princeton University, the Spanish Participation Group, University of Tokyo, University of Utah, Vanderbilt University, University of Virginia, University of Washington, and Yale University.

\section{Appendix A: The catalogues}

The catalogue of flux- and volume-limited groups and clusters of galaxies consists of several tables: two tables for each sample. The first table lists the galaxies that were used to generate the catalogue of groups and clusters, and the second one describes the group properties. The flux-limited galaxy table includes all the relevant parameters for galaxies. The volume-limited galaxy table includes only the basic parameters and references to the flux-limited sample. The structure of group catalogues for the flux- and volume-limited samples is identical.

The catalogues are available at http://cosmodb.to. ee. The catalogues will be made available also through the Strasbourg Astronomical Data Centre (CDS).

\section{A.1. Description of the flux-limited galaxy catalogue}

The flux-limited galaxy catalogue contains the following information (column numbers are given in square brackets):

1. [1] galid - our unique identification number for galaxies;

2. [2] specobjid - SDSS DR10 spectroscopic object identification number;

3. [3] objid - SDSS DR10 photometric object identification number;

4. [4] groupid - group/cluster id;
5. [5] ngal - richness (number of members) of the group/cluster the galaxy belongs to;

6. [6] rank - luminosity rank of the galaxy within its group; rank 1 indicates the most luminous galaxy;

7. [7] groupdist - comoving distance to the group/cluster centre to which the galaxy belongs, in units of $h^{-1} \mathrm{Mpc}$, calculated as an average over all galaxies within the group/cluster;

8. [8] zobs - observed redshift (without the CMB correction), as given in the SDSS CAS;

9. [9] zcmb - redshift, corrected to the CMB rest frame;

10. [10] zerr - uncertainty of the redshift;

11. [11] dist - comoving distance in units of $h^{-1} \mathrm{Mpc}$ (calculated directly from the CMB-corrected redshift);

12. [12] dist_cor - comoving distance of the galaxy after suppressing of the finger-of-god effect (as used in the luminosity density field calculations);

13. [13-14] raj2000, dej2000 - right ascension and declination (deg);

14. [15-16] glon, glat-galactic longitude and latitude (deg);

15. [17-18] sglon, sglat - supergalactic longitude and latitude (deg);

16. [19-20] lam, eta - SDSS survey coordinates $\lambda$ and $\eta$ (deg);

17. [21-23] crd_xyz - cartesian coordinates defined by $\eta$ and $\lambda$;

18. [24-28] mag_x - Galactic-extinction-corrected Petrosian magnitude ( $x \in$ ugriz filters);

19. [29-33] absmag_x - absolute magnitude of the galaxy, $k+e$ corrected ( $x \in$ ugriz filters, in units of mag $+5 \log _{10} h$ );

20. [34-38] kecor_x $-k+e$-correction ( $x \in$ ugriz filters);

21. [39-43] ext_x Galactic extinction ( $x \in$ ugriz filters);

22. [44] lum_r - observed luminosity in the $r$-band in units of $10^{10} h^{-2} L_{\odot}$, where $M_{\odot}=4.64$ (Blanton \& Roweis 2007);

23. [45] weight - weight factor for the galaxy (w.lum_r was used to calculate the luminosity density field);

24. [46] source - source of the redshift: 0 for SDSS, 1 for 2MRS, 2 for $2 \mathrm{dFGRS}, 3$ for RC3;

25. [47] bad_lum - if set to 1, galaxy luminosity is uncertain;

26. [48] morf - morphology of the galaxy $(0-$ unclear, $1-$ spiral, 2 - elliptical);

27. [49] morf_zoo - morphology from the galaxy zoo project (Lintott et al. 2008): 0 - unclear, 1 - spiral, 2 - elliptical;

28. [50] hc_e - probability of being an early-type galaxy (from Huertas-Company et al. 2011);

29. [51] hc_s0 - probability of being an S0 galaxy;

30. [52] hc_sab - probability of being an Sab galaxy;

31. [53] hc_scd - probability of being an Scd galaxy;

32. [54] dist_edge - comoving distance of the galaxy from the border of the survey mask;

33. [55-58] den $a-$ normalised environmental density of the galaxy for various smoothing scales $\left(a=1,2,4,8 h^{-1} \mathrm{Mpc}\right)$.

\section{A.2. Description of the volume-limited galaxy catalogue}

The volume-limited galaxy catalogues contain the following information (column numbers are given in square brackets):

1. [1] galid - our unique identification number for galaxies (identical with the flux-limited galaxy identification number);

2. [2] groupid - group/cluster id, unique within one volumelimited sample;

3. [3] ngal - richness (number of members) of the group the galaxy belongs to; 
4. [4] rank - luminosity rank of the galaxy within its group; rank 1 indicates the most luminous galaxy;

5. [5-9] absmag_x - absolute magnitude of the galaxy, $k+e$ corrected ( $x \in$ ugriz filters, in units of mag $+5 \log _{10} h$ );

6. [10] lum_r - observed luminosity in the $r$-band in units of $10^{10} h^{-2} L_{\odot}$, where $M_{\odot}=4.64$ (Blanton \& Roweis 2007);

7. [11] dist_cor - comoving distance of the galaxy after suppressing the finger-of-god effect;

8. [12-14] crd_xyz - cartesian coordinates defined by $\eta$ and $\lambda$.

\section{A.3. Description of the group/cluster catalogues}

The catalogue of groups/clusters contains the following information (column numbers are given in square brackets):

1. [1] groupid - group/cluster id;

2. [2] ngal - richness (number of members) of the group;

3. [3-4] raj2000, dej2000 - right ascension and declination of the group centre (deg);

4. [5] zcmb - CMB-corrected redshift of group, calculated as an average over all group/cluster members;

5. [6] groupdist - comoving distance to the group centre $\left(h^{-1} \mathrm{Mpc}\right)$;

6. [7] sigma_v - rms radial velocity deviation ( $\sigma_{V}$ in physical coordinates, in $\mathrm{km} \mathrm{s}^{-1}$ );

7. [8] sigma_sky - rms deviation of the projected distance in the sky from the group centre $\left(\sigma_{\text {sky }}\right.$ in physical coordinates, in $\left.h^{-1} \mathrm{Mpc}\right), \sigma_{\text {sky }}$ defines the extent of the group in the sky;

8. [9] r_vir - virial radius in $h^{-1} \mathrm{Mpc}$ (the projected harmonic mean, in physical coordinates);

9. [10] r_max - maximum radius of the group/cluster;

10. [11] mass_nfw - estimated mass of the group assuming the NFW density profile (in units of $10^{12} h^{-1} M_{\odot}$ );

11. [12] mass_her - estimated mass of the group assuming the Hernquist density profile (in units of $10^{12} h^{-1} M_{\odot}$ );

12. [13] lum_r_group - observed luminosity, i.e. the sum of the luminosities of the galaxies in the group/cluster $\left(10^{10} h^{-2} L_{\odot}\right)$;

13. [14] weight - weight factor for the group at the mean distance of the group;

14. [15-18] den $a$ - normalised environmental density (mean of group galaxy densities) of the group for various smoothing scales $\left(a=1,2,4,8 h^{-1} \mathrm{Mpc}\right)$.

\section{A.4. Access to the SDSS database}

To facilitate the use of all the parameters available in the SDSS CAS, we have uploaded our galaxy galid-s together with the SDSS objid and specobjid to the CAS server. For example, to add a Galactic-extinction-corrected model magnitude in the $r$-band (dered_r) from the SDSS CAS to our catalogue, the following SQL query in CAS can be used:

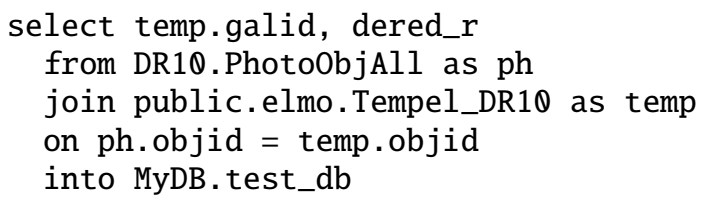

\section{Appendix B: Estimation of galaxy luminosity evolution}

Galaxy luminosity varies over time. Because of the secular evolution of galaxies, their luminosity slightly increases over time,

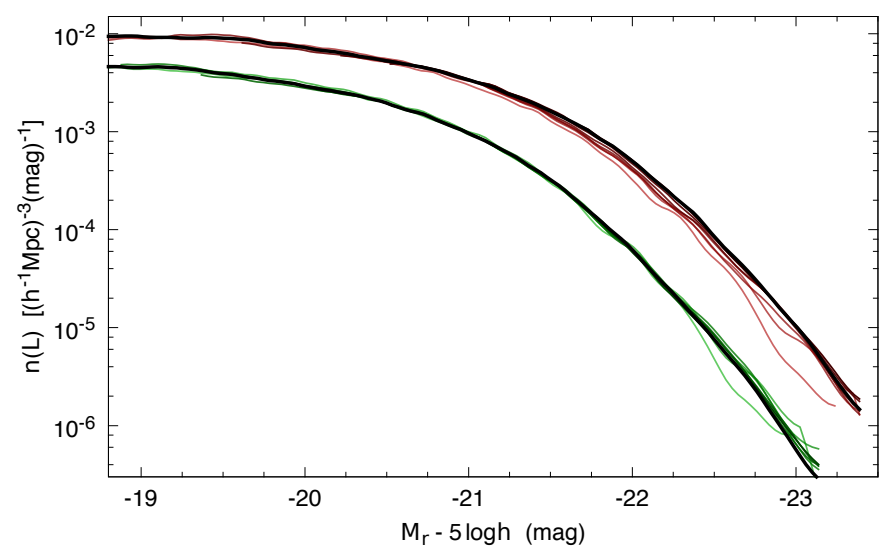

Fig. B.1. Upper lines show the luminosity function for various distance intervals (see text for details) without evolution corrections. Lower lines show the luminosity function with evolution correction.

even if no major influence affects their evolution. For a statistical analysis this small luminosity evolution has to be taken into account.

The luminosity evolution of the SDSS galaxies was estimated in Blanton et al. (2003), who basically assumed that the luminosity function of galaxies is constant with distance. In practice, the luminosity evolution is different for various types of galaxies. For simplicity, Blanton et al. (2003) assumed that the luminosity evolution for the SDSS main sample galaxies (a relatively nearby region) can be described using the equation $e_{\text {cor }}=Q z$, where $Q$ is some constant and $z$ is the redshift (Eq. (1) shows how the correction is applied). Blanton et al. (2003) estimated the constant for the $r$-filter to be -1.62 . The estimate of this constant is based on the early-data release of the SDSS.

Below, we used the same simple approach and re-estimated the luminosity evolution of galaxies from the most recent dataset, SDSS DR10. To estimate the luminosity evolution, we calculated the luminosity function in eight distance intervals centred at 125, 175, 225, 275, 325, 375, 425, and $475 \mathrm{~h}^{-1} \mathrm{Mpc}$ : the distance regions are $\pm 75 h^{-1} \mathrm{Mpc}$. By changing the luminosity evolution parameter $Q$, we found the value that produces most similar luminosity functions at different distance intervals. For that we calculated the sum of the relative differences between the luminosity function in one distance interval and the luminosity function measured using all galaxies. Figure B.1 shows the luminosity function for the $r$ filter for two cases. The upper set of lines shows the luminosity function without the evolution correction, the lower set of lines shows the luminosity functions in various distance intervals with evolution corrections. After the correction, the luminosity functions in various distance intervals are roughly the same. Our estimated evolution corrections for the $r$-filter is -1.10 , which is slightly smaller than given by Blanton et al. (2003).

\section{Appendix C: Density fields}

To estimate the environmental densities for galaxies and groups, we calculated the smoothed luminosity density field. Details of the calculation of the density fields are given in Liivamägi et al. (2012). For consistency reasons, a brief description is given below.

Assuming that in addition to the observed galaxies, each group may also contain galaxies that lie outside of the observational window of the survey, the estimated total luminosity per 
one visible galaxy is

$L_{\mathrm{tot}}=L_{\mathrm{obs}} \cdot W_{d}$

where $L_{\mathrm{obs}}$ is the observed luminosity of the galaxy. Thus the luminosity $L_{\text {tot }}$ takes into account the expected luminosities of the unobserved galaxies. It can, for example, be used for calculating the full luminosity density field. However, it cannot be used to estimate the total luminosity of any particular group since the luminosity function of galaxies depends on group properties.

The distance-dependent weight factor $W_{d}$ is defined as

$W_{d}=\frac{\int_{0}^{\infty} \operatorname{Ln}(L) \mathrm{d} L}{\int_{L_{1}}^{L_{2}} \operatorname{Ln}(L) \mathrm{d} L}$,

where $L_{1,2}=L_{\odot} 10^{0.4\left(M_{\odot}-M_{1,2}\right)}$ are the luminosity limits of the observational window at the distance $d$ and $n(L)$ is luminosity function. The weight factor as a function of distance is given in the inner panel of Fig. 2.

To determine the luminosity density field, we used a kernel sum:

$\ell_{\mathbf{i}}=\frac{1}{a^{3}} \sum_{\mathrm{gal}} K^{(3)}\left(\frac{\boldsymbol{r}_{\mathrm{gal}}-\boldsymbol{r}_{\mathbf{i}}}{a}\right) L_{\mathrm{tot}}$,

where $L_{\text {tot }}$ is the weighted galaxy luminosity and $a$ - the kernel scale. For the kernel $K(\cdot)$ we used the $B_{3}$ spline function:

$B_{3}(x)=\frac{|x-2|^{3}-4|x-1|^{3}+6|x|^{3}-4|x+1|^{3}+|x+2|^{3}}{12}$.

The luminosity density field was calculated on a regular cartesian grid generated by using the SDSS $\eta$ and $\lambda$ angular coordinates. This allows the most efficient placing of the cone inside a brick.

While calculating the density field, we also suppressed the finger-of-god redshift distortions using the rms sizes of galaxy groups in the sky, $\sigma_{\text {sky }}$, and their rms radial velocities, $\sigma_{v}$, (both in physical coordinates at the location of the group). For that, we calculated the new radial distances for galaxies $\left(d_{\text {gal }}\right)$ as

$d_{\text {gal }}=d_{\text {group }}+\left(d_{\text {gal }}^{\star}-d_{\text {group }}\right) \frac{\sigma_{\text {sky }}}{\sigma_{v} / H_{0}}$,

where $d_{\text {gal }}^{\star}$ is the initial distance to the galaxy, and $d_{\text {group }}$ is the distance to the group centre. For double galaxies, where the extent of the system in the plane of the sky does not have to show its real size (because of projection effects), we demanded that its (comoving) size along the line-of-sight does not exceed the comoving LL, $d_{\mathrm{LL}}(z)$, used to define the system

$d_{\text {gal }}=d_{\text {group }}+\left(d_{\text {gal }}^{\star}-d_{\text {group }}\right) \frac{d_{\mathrm{LL}}(z)}{\left|v_{1}-v_{2}\right| / H_{0}}$,

if $\left|v_{1}-v_{2}\right| / H_{0}>d_{\mathrm{LL}}(z)$.

Here $z$ is the mean redshift of the double system. If the velocity difference is smaller than that quoted above, we did not change the galaxy distances.

We analysed the difference between the $B_{3}$ spline kernel and the Gaussian kernel. The kernels are plotted in Fig. C.1, showing that the general shape of these kernels is very similar, but the $B_{3}$ spline kernel drops to zero behind 2.0, thus it does not extend to infinity. In addition, the $B_{3}$ spline kernel is the most compact set of polynomials for a given degree, and they are interpolating,

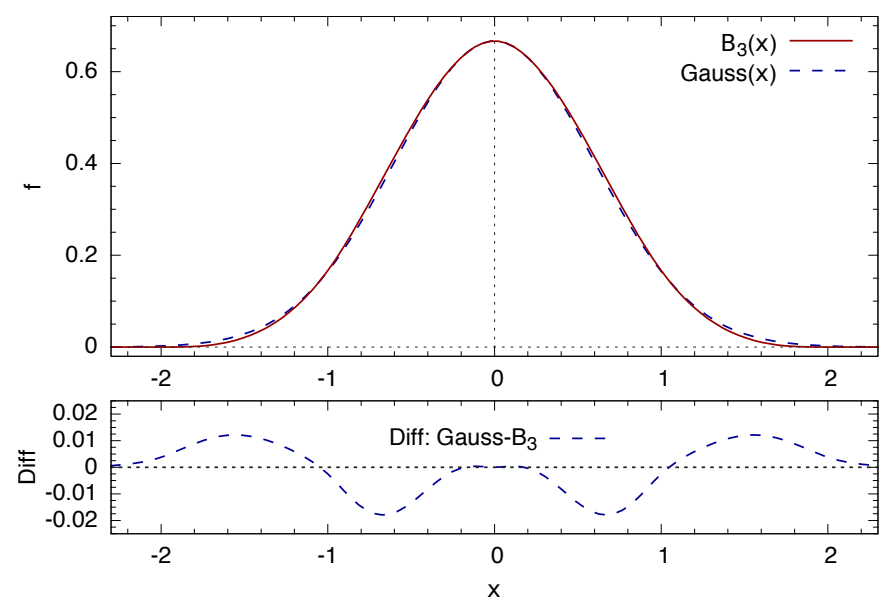

Fig. C.1. Gaussian (dashed line) and $B_{3}$ spline (solid line) kernels. Here, the $B_{3}$ kernel has $a=1.0$ and the Gaussian kernel has $\sigma=0.5984$. The Gaussian standard deviation $\sigma$ is chosen to have the same central density as the $B_{3}$ kernel. The lower panel shows the difference between the two kernels, scaled to the central density.

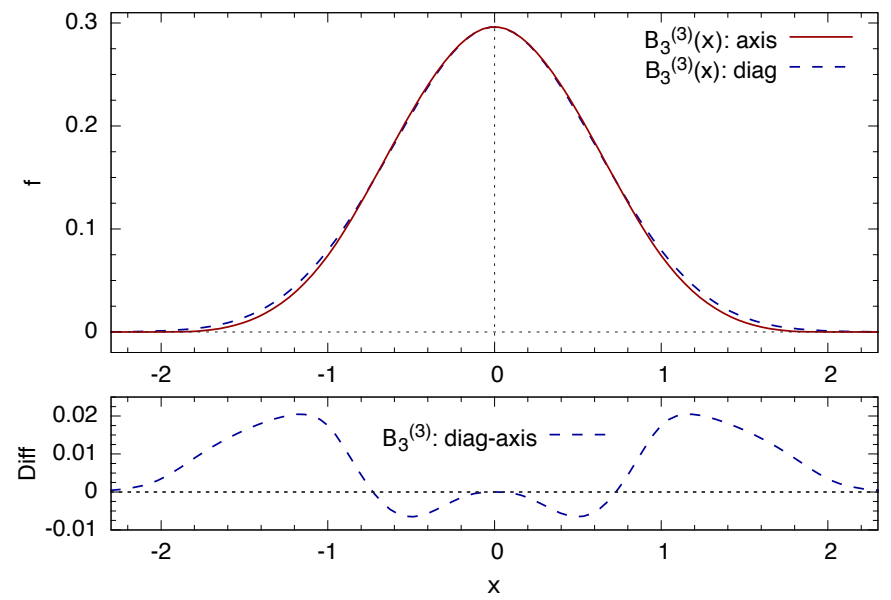

Fig. C.2. Deviation from isotropy for the $3 \mathrm{D} B_{3}$ spline kernel. Upper panel shows the $B_{3}$ distribution for two extreme cases: along the coordinate axis and along the diagonal of the box. The lower panel shows the difference (in units of the central density) between these extremes. The maximum deviation from isotropy is lower than two per cent.

meaning that their sum over a grid is always unity. This aspect makes them ideal smoothing kernels.

Equation (C.4) implies that the three-dimensional $B_{3}$ kernel is not perfectly isotropic. The deviation for extreme cases is shown in Fig. C.2. The maximum deviation is lower than $2 \%$ of the central density. Within most of the volume covered by $B_{3}$, the deviation remains far lower than $1 \%$. Thus the anisotropy has a negligible effect when using the kernel for density field calculations.

Next, we compared the density fields generated using the $B_{3}$ kernel and the Gaussian kernel. For comparison, we fixed $a=$ $1.0 h^{-1} \mathrm{Mpc}$ for the $B_{3}$ kernel and $\sigma=0.5984$ for the Gaussian kernel, which yielded the same central density. Figure C.3 shows the relative difference between the resulting density fields. In high-density regions (more than 10 times the average density), the differences are rather small. However, in low-density regions, the differences can be very high. This is expected, since Gaussian kernels extend to infinity and the density field of low-density regions becomes affected by galaxies that are also located in denser environments. 


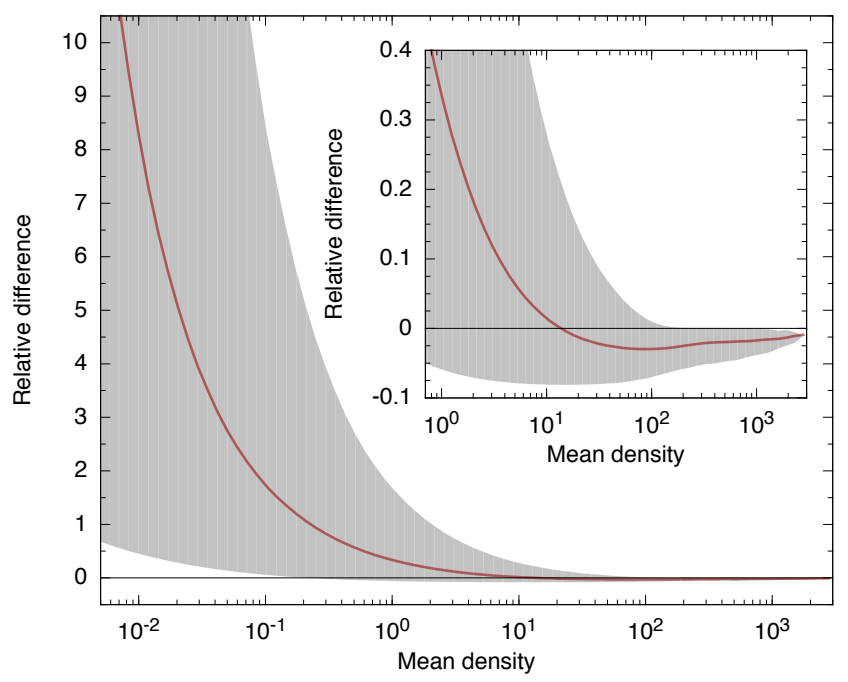

Fig. C.3. Relative difference $\left(\left(\ell_{\text {Gauss }}-\ell_{B_{3}}\right) / \ell_{\text {Gauss }}\right)$ between luminosity density fields smoothed with the Gaussian kernel and with the $B_{3}$ spline kernel. Density is scaled to the mean density.

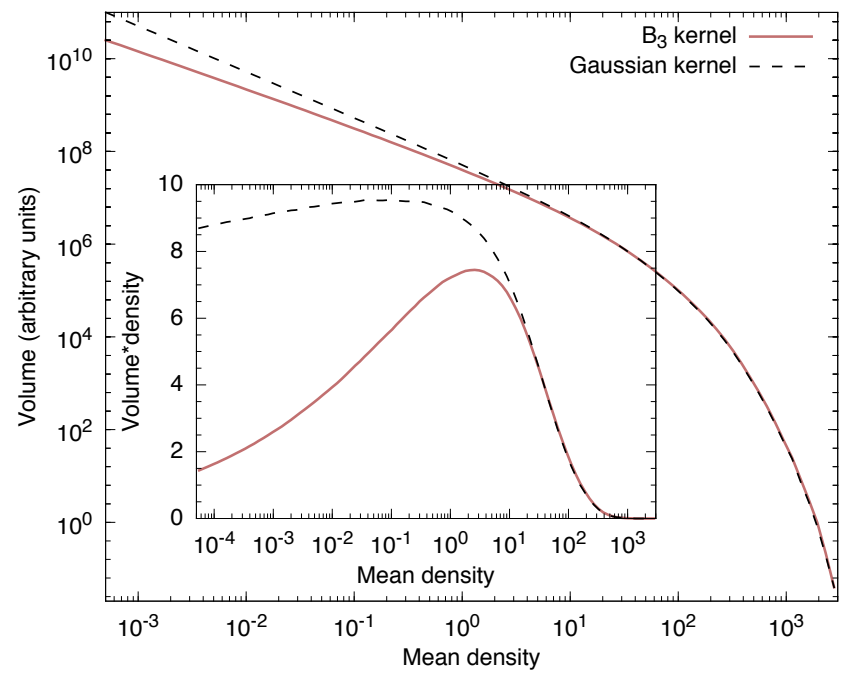

Fig. C.4. Number of cells for a fixed density interval. The main panel shows the number of cells for linear density bins and the inset panel those for logarithmic density bins. The density is scaled to the mean density.

Figure C.4 gives the number of cells for a fixed density interval for linear (outer panel) and logarithmic (inner panel) density bins. The volume covered by high-density regions (more than 10 times the average density) is practically the same. For lower density environments, the volume for Gaussian smoothing is a few times larger than for $B_{3}$ smoothing, which distorts the density distribution in low-density environments.

To summarise, the comparison of the Gaussian and $B_{3}$ kernels indicates that for high-density environments, the results are practically the same, while for lower density environments, the differences become very large. Using the Gaussian smoothing, the densities in low-density environments are much higher than for the $B_{3}$ kernels. Saar (2009) has also shown that the Minkowski functionals are very noisy and untrustworthy in low-density regions.

\section{References}

Abazajian, K. N., Adelman-McCarthy, J. K., Agüeros, M. A., et al. 2009, ApJS, 182,543

Ahn, C. P., Alexandroff, R., Allende Prieto, C., et al. 2012, ApJS, 203, 21

Ahn, C. P., Alexandroff, R., Allende Prieto, C., et al. 2014, ApJS, 211, 17

Aihara, H., Allende Prieto, C., An, D., et al. 2011, ApJS, 193, 29

Alpaslan, M., Robotham, A. S. G., Driver, S., et al. 2014, MNRAS, 438, 177

Bahcall, N. A., Dong, F., Bode, P., et al. 2003, ApJ, 585, 182

Bartelmann, M. 1996, A\&A, 313, 697

Berlind, A. A., Frieman, J., Weinberg, D. H., et al. 2006, ApJS, 167, 1

Binney, J., \& Tremaine, S. 2008, Galactic Dynamics, 2nd edn. (Princeton University Press)

Blanton, M. R., \& Roweis, S. 2007, AJ, 133, 734

Blanton, M. R., Hogg, D. W., Bahcall, N. A., et al. 2003, ApJ, 592, 819

Budzynski, J. M., Koposov, S. E., McCarthy, I. G., \& Belokurov, V. 2014, MNRAS, 437, 1362

Carollo, C. M., Cibinel, A., Lilly, S. J., et al. 2013, ApJ, 776, 71

Catinella, B., Schiminovich, D., Cortese, L., et al. 2013, MNRAS, 436, 34

Choi, Y.-Y., Han, D.-H., \& Kim, S. S. 2010, J. Korean Astron. Soc., 43, 191

Colless, M., Dalton, G., Maddox, S., et al. 2001, MNRAS, 328, 1039

Colless, M., Peterson, B. A., Jackson, C., et al. 2003 [arXiv: astro-ph/0306581]

Corwin, Jr., H. G., Buta, R. J., \& de Vaucouleurs, G. 1994, AJ, 108, 2128

Davis, M., \& Geller, M. J. 1976, ApJ, 208, 13

de Vaucouleurs, G., de Vaucouleurs, A., Corwin, Jr., H. G., et al. 1991, Third Reference Catalogue of Bright Galaxies, Vols I-III (New York: Springer)

Dressler, A. 1980, ApJ, 236, 351

Duarte, M., \& Mamon, G. A. 2014, MNRAS, 440, 1763

Einasto, J., Saar, E., Kaasik, A., \& Chernin, A. D. 1974, Nature, 252, 111

Einasto, J., Hütsi, G., Einasto, M., et al. 2003, A\&A, 405, 425

Einasto, M., Einasto, J., Tago, E., Dalton, G. B., \& Andernach, H. 1994 MNRAS, 269, 301

Einasto, M., Einasto, J., Tago, E., Müller, V., \& Andernach, H. 2001, AJ, 122, 2222

Einasto, M., Saar, E., Martínez, V. J., et al. 2008, ApJ, 685, 83

Einasto, M., Liivamägi, L. J., Tempel, E., et al. 2011, ApJ, 736, 51

Einasto, M., Liivamägi, L. J., Tempel, E., et al. 2012, A\&A, 542, A36

Einasto, M., Lietzen, H., Tempel, E., et al. 2014, A\&A, 562, A87

Eke, V. R., Baugh, C. M., Cole, S., et al. 2004, MNRAS, 348, 866

Gal, R. R. 2006, unpublished [arXiv: astro-ph/0601195]

Hao, J., McKay, T. A., Koester, B. P., et al. 2010, ApJS, 191, 254

Hearin, A. P., Zentner, A. R., Newman, J. A., \& Berlind, A. A. 2013, MNRAS, 430, 1238

Hernquist, L. 1990, ApJ, 356, 359

Hess, K. M., \& Wilcots, E. M. 2013, AJ, 146, 124

Hou, A., Parker, L. C., Balogh, M. L., et al. 2013, MNRAS, 435, 1715

Huchra, J. P., Macri, L. M., Masters, K. L., et al. 2012, ApJS, 199, 26

Huertas-Company, M., Aguerri, J. A. L., Bernardi, M., Mei, S., \& Sánchez Almeida, J. 2011, A\&A, 525, A157

Huertas-Company, M., Shankar, F., Mei, S., et al. 2013, ApJ, 779, 29

Jackson, J. C. 1972, MNRAS, 156, 1

Jarrett, T. H., Chester, T., Cutri, R., Schneider, S. E., \& Huchra, J. P. 2003, AJ, 125,525

Kaviraj, S. 2014, MNRAS, 437, L41

Koester, B. P., McKay, T. A., Annis, J., et al. 2007, ApJ, 660, 239

Komatsu, E., Smith, K. M., Dunkley, J., et al. 2011, ApJS, 192, 18

Krause, M. O., Ribeiro, A. L. B., \& Lopes, P. A. A. 2013, A\&A, 551, A143

Lacerna, I., Rodriguez-Puebla, A., Avila-Reese, V., \& Hernandez-Toledo, H. M. 2014, ApJ, 788, 29

Lackner, C. N., \& Gunn, J. E. 2013, MNRAS, 428, 2141

Li, R., Shan, H., Mo, H., et al. 2014, MNRAS, 438, 2864

Lietzen, H., Tempel, E., Heinämäki, P., et al. 2012, A\&A, 545, A104

Liivamägi, L. J., Tempel, E., \& Saar, E. 2012, A\&A, 539, A80

Lintott, C. J., Schawinski, K., Slosar, A., et al. 2008, MNRAS, 389, 1179

Łokas, E. L., \& Mamon, G. A. 2001, MNRAS, 321, 155

Luparello, H. E., Lares, M., Yaryura, C. Y., et al. 2013, MNRAS, 432, 1367

Macciò, A. V., Dutton, A. A., \& van den Bosch, F. C. 2008, MNRAS, 391, 1940

Makarov, D., \& Karachentsev, I. 2011, MNRAS, 412, 2498

Martínez, V. J., Arnalte-Mur, P., Saar, E., et al. 2009, ApJ, 696, L93

McGee, S. L. 2013, MNRAS, 436, 2708

Montero-Dorta, A. D., \& Prada, F. 2009, MNRAS, 399, 1106

Muñoz-Cuartas, J. C., \& Müller, V. 2012, MNRAS, 423, 1583

Navarro, J. F., Frenk, C. S., \& White, S. D. M. 1997, ApJ, 490, 493

Nurmi, P., Heinämäki, P., Sepp, T., et al. 2013, MNRAS, 436, 380

Oemler, Jr., A. 1974, ApJ, 194, 1

Old, L., Gray, M. E., \& Pearce, F. R. 2013, MNRAS, 434, 2606

Old, L., Skibba, R. A., Pearce, F. R., et al. 2014, MNRAS, 441, 1513 
A\&A 566, A1 (2014)

Peng, Y.-J., \& Maiolino, R. 2014, MNRAS, 438, 262

Postman, M., \& Geller, M. J. 1984, ApJ, 281, 95

Press, W. H., \& Davis, M. 1982, ApJ, 259, 449

Reiprich, T. H., \& Böhringer, H. 2002, ApJ, 567, 716

Rines, K., \& Diaferio, A. 2006, AJ, 132, 1275

Rines, K., Diaferio, A., \& Natarajan, P. 2007, ApJ, 657, 183

Robotham, A. S. G., Norberg, P., Driver, S. P., et al. 2011, MNRAS, 416, 2640

Saar, E. 2009, in Data Analysis in Cosmology, ed. V. J. Martínez, E. Saar,

E. Martínez-González, \& M.-J. Pons-Bordería, (Berlin: Springer Verlag),

Lect. Notes Phys., 665, 523

Schlegel, D. J., Finkbeiner, D. P., \& Davis, M. 1998, ApJ, 500, 525

Skrutskie, M. F., Cutri, R. M., Stiening, R., et al. 2006, AJ, 131, 1163

Strauss, M. A., Weinberg, D. H., Lupton, R. H., et al. 2002, AJ, 124, 1810

Tago, E., Einasto, J., Saar, E., et al. 2006, Astron. Nachr., 327, 365

Tago, E., Einasto, J., Saar, E., et al. 2008, A\&A, 479, 927

Tago, E., Saar, E., Tempel, E., et al. 2010, A\&A, 514, A102

Tempel, E., Einasto, J., Einasto, M., Saar, E., \& Tago, E. 2009, A\&A, 495, 37

Tempel, E., Saar, E., Liivamägi, L. J., et al. 2011, A\&A, 529, A53
Tempel, E., Tago, E., \& Liivamägi, L. J. 2012, A\&A, 540, A106

Tempel, E., Stoica, R. S., Martínez, V. J., et al. 2014, MNRAS, 438, 3465

Tovmassian, H. M., \& Plionis, M. 2009, ApJ, 696, 1441

Tucker, D. L., Oemler, Jr., A., Hashimoto, Y., et al. 2000, ApJS, 130, 237

Tully, R. B., \& Fisher, J. R. 1978, in Large Scale Structures in the Universe, eds.

M. S. Longair, \& J. Einasto, IAU Symp., 79, 31

Turner, E. L., \& Gott, III, J. R. 1976, ApJS, 32, 409

Wen, Z. L., Han, J. L., \& Liu, F. S. 2012, ApJS, 199, 34

Wetzel, A. R., Tinker, J. L., Conroy, C., \& van den Bosch, F. C. 2013, MNRAS 432, 336

Wojtak, R. 2013, A\&A, 559, A89

Yang, X., Mo, H. J., van den Bosch, F. C., \& Jing, Y. P. 2005, MNRAS, 356, 1293

Yang, X., Mo, H. J., van den Bosch, F. C., et al. 2007, ApJ, 671, 153

Yang, X., Mo, H. J., van den Bosch, F. C., et al. 2013, ApJ, 770, 115

York, D. G., Adelman, J., Anderson, Jr., J. E., et al. 2000, AJ, 120, 1579

Zhang, Y., Yang, X., Wang, H., et al. 2013, ApJ, 779, 160

Zucca, E., Zamorani, G., Scaramella, R., \& Vettolani, G. 1993, ApJ, 407, 470 